\title{
Investigation of the \\ American Petroleum Institute's \\ Support Stiffness Ratio Threshold \\ Specification
}

\author{
A Thesis Presented to the faculty of \\ the School of Engineering and Applied Science \\ University of Virginia
}

in partial fulfillment

of the requirements for the degree

Master of Science

by

David Jeter Griffin Jr.

August 2016 
The Thesis

is submitted in partial fulfillment of the requirements

for the degree of

Master of Science

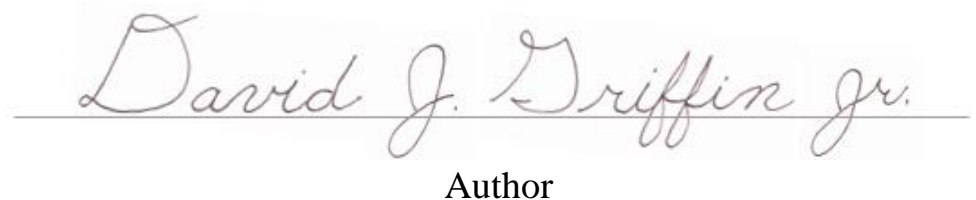

The thesis has been read and approved by the examining committee:

\author{
Dr. Chris P. Goyne \\ Advisor \\ Dr. Houston G. Wood \\ Dr. Roger L. Fittro \\ Dr. Jason R. Kerrigan
}

Accepted for the school of Engineering and Applied Science:

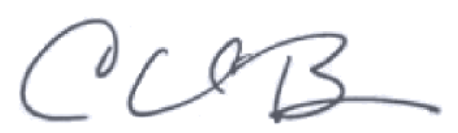

Craig H. Benson, Dean, School of Engineering and Applied Science

August 2016 


\begin{abstract}
In the design phase of developing a rotating machine, it is important to consider the effect that the supporting structure has on the rotordynamic behavior of the system. The American Petroleum Institute's (API) Standard Paragraphs state that if the support stiffness is greater than 3.5 times the bearing oil film stiffness, the designer may omit the supports from the rotordynamic analysis. As discussed in this paper, there is concern that machines operating near the second critical speed may not be adequately modeled using the rigid support assumption allowed by API. Due to the suspected influence of support dynamics on problem machines in industry, this paper investigates the effectiveness of the support to bearing stiffness ratio threshold of 3.5. A Jeffcott rotor model is used to capture the effect of the support on the separation margin and amplification factors of the first and second critical speeds. The trends are then validated using numerical models of two case studies. Results show that the API support stiffness ratio of 3.5 has limitations as a rigid support threshold and should be used with caution when the second critical speed is near a separation margin boundary. The paper proposes an addendum to the current API specification intended to reduce the risk of separation margin encroachment due to a rigid support assumption. Unlike other papers discussing the influence of flexible supports on rotating machinery, this investigation studies the effects at API's support stiffness ratio of 3.5 and compares them to a rigid support system.
\end{abstract}

Keywords: API standard, stiffness ratio, flexible support, rotordynamics 


\section{Acknowledgments}

The author would like to acknowledge Dr. Roger L. Fittro, Dr. Robert D. Rockwell, and Dr. Chris P. Goyne for their time and guidance during this investigation.

The author would like to thank Dr. C. Hunter Cloud and Dr. José A. Vázquez of BRG Machinery Consulting LLC for their input during this investigation.

The author would like to acknowledge the Rotating Machinery and Controls Laboratory at the University of Virginia, which supplied the funding and resources for the work described in this paper. 


\section{CONTENTS}

$1 \quad$ Introduction . . . . . . . . . . . . . . . . . . 1

2 Background and nomenclature . . . . . . . . . . . . . 4

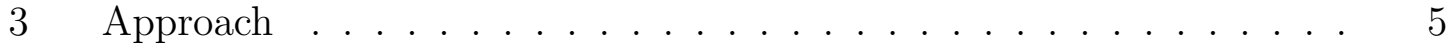

3.1 One-degree-of-freedom model $\ldots \ldots \ldots \ldots$

3.2 Two-degree-of-freedom model . . . . . . . . . . . . . 7

3.3 Jeffcott rotor model . . . . . . . . . . . . . . . . . . 7

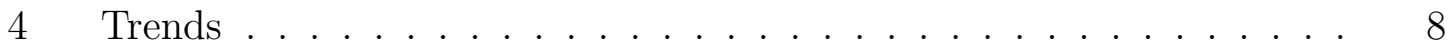

4.1 One-degree-of-freedom model . . . . . . . . . . . . 8

$4.2 \quad$ Jeffcott rotor model . . . . . . . . . . . . . . . . . . . . 10

$4.2 .1 \quad$ Unbalance response analysis . . . . . . . . . . . 11

4.2.2 Damped eigenvalue analysis . . . . . . . . . . 12

$4.2 .3 \quad$ Rotor stiffness ratio trends . . . . . . . . . . . . 13

4.2.4 Bearing parameter variation trends . . . . . . . . 14

5 Case study validation . . . . . . . . . . . . . . . . . . 17

$5.1 \quad$ Steam turbine case study . . . . . . . . . . . . . . 18

5.1.1 First mode unbalance response . . . . . . . . . 19

5.1.2 Second mode unbalance response . . . . . . . . . 20

5.1.3 Bearing parameter variation . . . . . . . . . 22

5.1.4 Damped eigenvalue analysis . . . . . . . . . 23

5.2 Centrifugal compressor case study . . . . . . . . . . 25

5.2 .1 Damped eigenvalue analysis . . . . . . . . . . 25

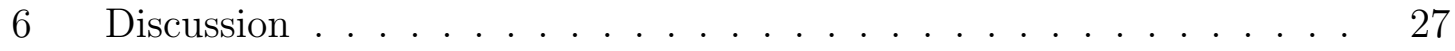


6.1 Questions of purpose ................ 27

6.2 Post-design support considerations . . . . . . . . . . . . . . 29

6.3 Support influence on subcritical machines _. . . . . . . . . . 30

6.4 Suggestion for API specification . . . . . . . . . . . . . 30

7 Conclusion . . . . . . . . . . . . . . . . . . . . 31

$\begin{array}{ll}\text { Appendix } & 37\end{array}$ 


\section{Introduction}

As turbomachinery is continuously pushed to achieve higher performance and efficiency, it becomes of paramount importance for the engineer to accurately predict the dynamic behavior of such systems in order to achieve a reliable and well-mannered machine. Specifications have been developed by the American Petroleum Institute (API) which, if adhered to, should ensure that the dynamic behavior of newly manufactured machines will be acceptable. One standard seen in many of the API specifications discusses the modeling of the support structure in the rotordynamic analysis of the turbomachine (API $6842^{\text {nd }}$ Edition 2005). The specification is as follows:

"The term "support" includes the foundation or support structure, the base, the machine frame and the bearing housing as appropriate. For machines whose bearing support system stiffness values are less than or equal to 3.5 times the bearing oil film stiffness values, support stiffness values derived from modal testing or calculated frequency dependent support stiffness and damping values (impedances) shall be used."

The interpretation of this standard suggests that if the support stiffness is greater than 3.5 times the bearing stiffness, the support may be assumed rigid (infinite stiffness). There are multiple reasons why this simplifying assumption is beneficial to the engineer. For example, in order to accurately model the supporting structure of turbomachinery, experimental data from the field, data from other similar machinery, or a computational model of the support structure is generally needed. This can be costly and time consuming to obtain (Vázquez and L. E. Barrett 1998). Often the support stiffness is unknown. In the case of a high volume product line, it would be unreasonable to expect an engineer to analyze every support structure on which a turbomachine is to be placed. By defining a threshold of the support to bearing stiffness ratio at 3.5, the engineer should be able to safely neglect the effect of the support 
on the dynamic behavior of the system for any machine that meets this criteria.

Although for many turbomachines this is a safe assumption, a concern arises when a machine is designed to operate just under the second critical speed. It is common for modern turbo machinery to operate above the first critical, and as higher speeds are necessary to achieve desired output requirements, operating speed ranges will continue to approach or reach the second critical (J. C. Nicholas 1989). As this investigation will confirm, the second critical speed is highly influenced by the support stiffness (J. Nicholas and L. Barrett 1986). Any assumptions in the prediction of the support dynamics can have a significant effect on the frequency and amplitude of the second critical speed response. In some cases this influence could be enough to lower the second critical into the separation margin region or even into the operating speed range of the machine. A machine running too close to a strong critical speed will result in increased vibration, higher bearing and rotor component forces, and an increased risk of losing critical machine clearances, ultimately leading to downtime or failure. Because of this concern, this investigation will highlight the circumstances where the support stiffness ratio of 3.5 is an appropriate threshold and where it may run into limitations.

The purpose of this paper is to answer the following questions:

1. What is the basis for the support stiffness ratio of 3.5 as a minimum?

2. What are the practical implications of the 3.5 support stiffness ratio?

3. What are some characteristics of a dynamic system where a support stiffness ratio of 3.5 becomes a relevant concern?

4. What classes or types of machinery have these dynamic properties?

5. Are there any better or additional guidelines that could be used to reduce or eliminate the above concerns? 
Many authors have analyzed the influence of bearing supports on turbomachinery. In 1925, Kimball found that the flexible support can influence the stability of a rotating system (Kimball Jr 1925). Lund concluded that rotor and support compliance lowers a system's stability threshold speed and when the support is damped, the compliant support can increase the stability threshold above that of a rigid support (Lund 1965). Kirk et al. also showed that a well-designed support system can increase the stability threshold speed of a rotating system (R. G. Kirk and E. J. Gunter 1972, 1975). Nicholas and Barrett (1986) showed that first and second critical speeds can be accurately predicted using a support stiffness and damper in series with a bearing stiffness and damper. They show that the support is highly influential on stiff second rotor modes, similar to the trends from this investigation (J. Nicholas and L. Barrett 1986). Later Nicholas related this work to the maturing versions of the API 612 (steam-turbine) specification (J. C. Nicholas 1989). In 2000 and 2001, Vázquez et al. published two papers regarding critical speed prediction and the effects of compliant supports on stability and unbalance response using numerical analysis and experimental data (Vázquez, L. E. Barrett, and Flack 2001a, b). Dikmen et al generated simple support models using modal analysis data and finite element models, the equivalent models were then used in the finite element model of the rotor to predict rotor response (Dikmen et al. 2009). Birchfield et al. analyzed the effects of coupling substructure dynamics with turbomachinery and the effect of the two on the system resonances. The authors investigated structural modifications based on the transfer functions of the structure alone in order to change the overall system's dynamic response (Birchfield, Singh, and Singhal 2013). Kang et al. published an investigation of the finite element modeling of substructures and their lumped mass inclusion in rotordynamics analyses. Numerical examples were used to suggest design criteria for avoiding resonances and suppressing vibration response (Kang et al. 2000). The aforementioned works are fundamental in analyzing the effects of flex- 
ible supports on rotating machinery, two critical aspects of which are the lowering of system natural frequencies and damping (Caruso, Gans, and Catlow 1982), both confirmed by this investigation. The work by Nicholas (1989) was similar in style to this investigation, demonstrating the effect of flexible supports on turbomachinery from an API specification standpoint, however the support stiffness ratio was not investigated.

Although the effects of compliant supports have been investigated in depth, no research to date has investigated the origin or adequacy of the API threshold ratio. This investigation will capture the risk of blindly assuming a rigid support assumption for a machine which meets the API support stiffness threshold. This is the first time such a study has been documented.

This study will discuss the influence of the API support stiffness ratio from an amplification factor and separation margin standpoint. First, a simplified model that captures the behavior of an actual turbomachine is identified. The damped eigenvalue and unbalance response trends from that model will be discussed. The simplified model trends will then be validated using two case studies. A discussion

and conclusions will follow to answer the five proposed questions and highlight the key points of the analysis. Finally, a revision to the current API support stiffness ratio specification will be suggested.

\section{Background and nomenclature}

In order to properly describe the simplified models, a few key terms need to be defined. The support stiffness ratio, $K$, is shown in Eqn. (1), where $k_{s}$ is the support stiffness and $k_{b}$ is the bearing stiffness. This is the ratio of concern for this investigation.

$$
K=\frac{k_{s}}{k_{b}}
$$


The rotor stiffness ratio, $R S R$, is defined by Eqn. (2), where $k_{r}$ is the rotor stiffness.

$$
R S R=\frac{2 k_{b}}{k_{r}}
$$

The bearing damping will be defined by Eqn. (3), where $\omega_{b}$ is the natural frequency of the rigid-rotor, rigid-support system (Eqn. 4), and $m$ is the rotor mass.

$$
\begin{gathered}
\zeta_{b}=\frac{c_{b}}{m \omega_{b}} \\
\omega_{b}=\sqrt{\frac{2 k_{b}}{m}}
\end{gathered}
$$

As mentioned in the previous section, this analysis will be approached from an amplification factor and separation margin perspective, meaning that the separation margins of the rigid and flexible support critical speeds will be compared, and trends based on the resulting separation margins will be discussed. Per the current API standards, any critical speed with an amplification factor of 2.5 or greater is a critical speed of concern and requires separation margins. The separation margins dictate how close to a critical speed a machine is allowed to operate. Any critical speed with an amplification factor of less than 2.5 is considered critically damped and no separation margin is required (API $6842^{\text {nd }}$ Edition 2005). A detailed explanation of amplification factor and separation margin may be found in Appendix A.

\section{Approach}

Although the recommended support stiffness ratio threshold has only been used by the API since 1996, its exact numerical significance has not been clearly documented (API $6841^{\text {st }}$ Edition 1996). Based on feedback from industry, it is understood that the threshold number of 3.5 could have been originally derived from a simple dynamic model based on some relationship of behavior between a rigid support and compliant 
support model. In an attempt to derive this number from a simple model, a series of models ranging from one to two degrees-of-freedom with varying non-dimensional stiffness and damping parameters were analyzed.

\subsection{One-degree-of-freedom model}

The simplest model was the single-degree-of-freedom system shown in Fig. 1. This model demonstrates the effect of adding a compliant support to a spring-mass-damper system; however, being a single-degree-of-freedom model, it cannot capture the effect of the support compliance on the second critical speed as seen in rotating machinery.

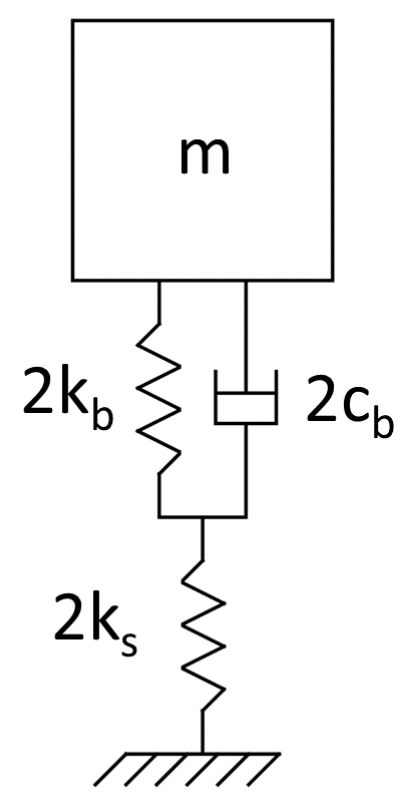

Fig. 1: Single-degree-of-freedom model used to show general trends from introduction of compliant support 


\subsection{Two-degree-of-freedom model}

A two-degree-of-freedom model, similar to the one seen in Fig. 1 was also analyzed, however the second degree of freedom was created by including the support mass. Although this model did generate two modes, the mode of concern in this investigation is the second rotor mode, not the support induced mode. Most API specifications (API 611, API 612, API 617, etc.) prohibit any structural support resonances within

the operating speed range and separation margins of the machine (API $6842^{\text {nd }}$ Edition 2005). Because of this specification and the fact that the API support stiffness ratio standard does not specify any mass or frequency dependency of the support, the support mass is omitted from the analyzed models and the trends from this model are not discussed further. More information on the behavior of this system can be found in Kirk and Gunter (1972).

\section{$3.3 \quad$ Jeffcott rotor model}

In order to better replicate a supercritical flexible rotor machine, a two-degree-offreedom Jeffcott rotor model was chosen as shown in Fig. 2. This model results in two rotor modes and will be the primary model for the analysis in this investigation. The two mode shapes are shown in Fig. 3. The first mode is a lateral "bounce" mode and the second is a conical tilting mode; these are typical mode shapes in turbomachinery. For the equations of motion for this model, refer to Appendix B.

It should be noted that all models used in this investigation assume zero support damping as support damping is typically very low relative to the bearing damping ( $\mathrm{J}$. Nicholas and L. Barrett 1986, R. G. Kirk and E. J. Gunter 1972). More information of the support damping effect can be seen in Appendix C. 


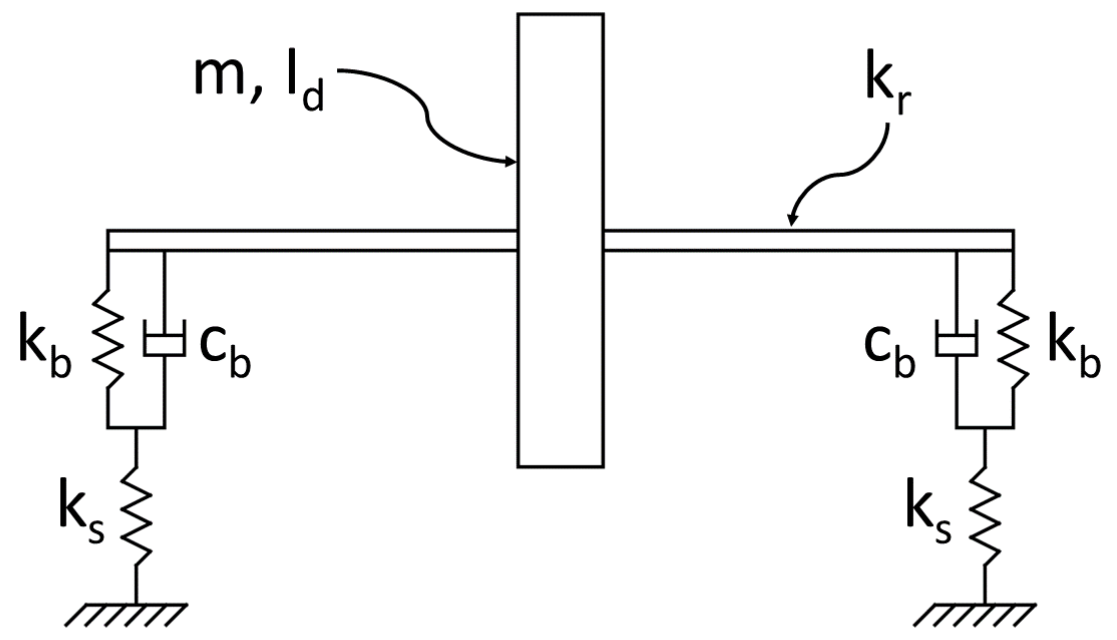

Fig. 2: Jeffcott model used to show trends from a two-mode system with compliant support. This model captures the influence of a compliant support on the dynamic behavior of machinery.

\section{Trends}

The trends of the one-degree-of-freedom model are examined first. Based on the insight gained, the trends of the more complicated Jeffcott rotor model are then presented in section 4.2. The effect of the support stiffness ratio on the behavior of the one-degree-of-freedom model is based on variation of the damped eigenvalues of the system. The effect of the support stiffness ratio on the behavior of the Jeffcott rotor model is based on both damped eigenvalue and the unbalance response analysis.

\subsection{One-degree-of-freedom model}

The simplest model (Fig. 1) was used to analyze the relation between a rigid support machine and a compliant support machine by calculating and comparing the equivalent system parameters for the two configurations. These equivalent parameters 


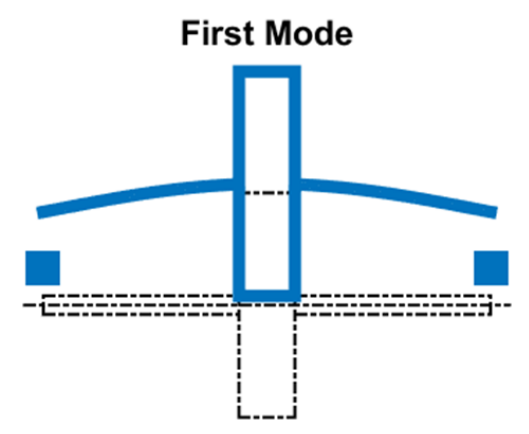

\section{Second Mode}

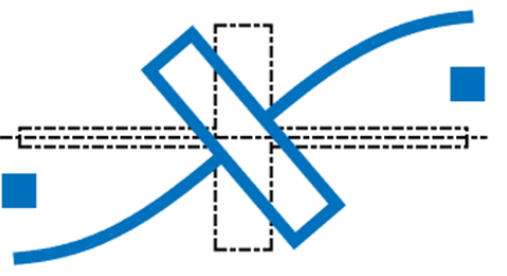

Fig. 3: Two modes resulting from compliant support Jeffcott rotor model

are shown in Fig. 4 and organized in Tab. 1.

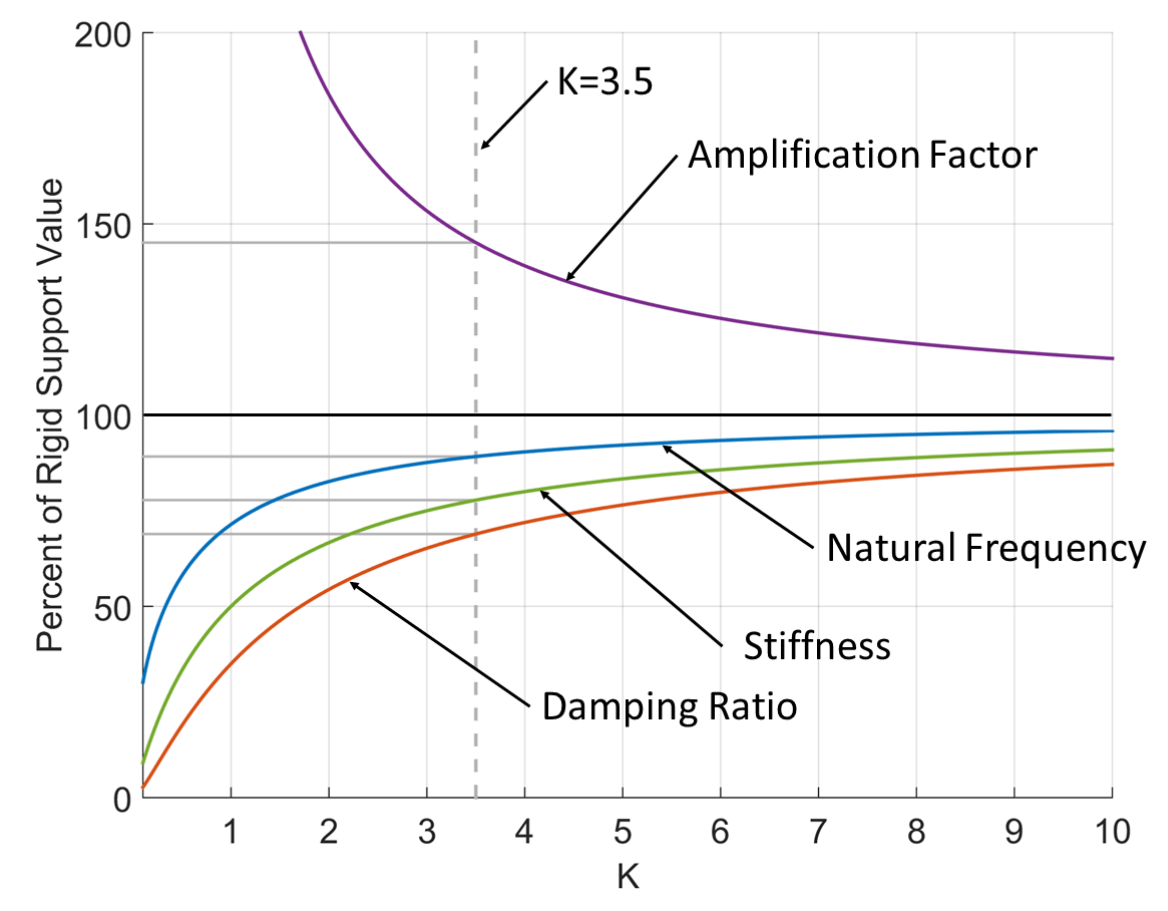

Fig. 4: The change in equivalent parameters as a percentage of the rigid support values for varying support stiffness ratios. $\zeta_{b}=0.2$

It is seen from the data in Tab. 1 that the natural frequency of a one-degree-offreedom model with a rigid support is $100 \%$. When adding a support with stiffness of $3.5 k_{b}(\mathrm{~K}=3.5)$ in series with $k_{b}$, the equivalent system natural frequency drops to about $89 \%$ of the rigid support system. The same interpretation is extended to the other results in Tab. 1. This analysis is useful to show that the introduction of 
Tab. 1: Equivalent system properties with compliant support $(\mathrm{K}=3.5)$ relative to rigid support model. $\zeta_{b}=0.2$

\begin{tabular}{lcc}
\hline Parameter & Rigid & Compliant: K=3.5 \\
\hline Natural Freq. & $100 \%$ & $89 \%$ \\
Stiffness & $100 \%$ & $78 \%$ \\
Damping & $100 \%$ & $69 \%$ \\
AF & $100 \%$ & $145 \%$ \\
\hline
\end{tabular}

a compliant support to a single-degree-of-freedom system acts to lower the effective natural frequency, the effective damping, and the effective stiffness of the system. Figure 4 shows that, at a support stiffness ratio of 3.5, the equivalent parameters are asymptotically approaching the rigid support values. The natural frequency, stiffness, damping, and amplification factor values are converging at a rate of $3 \% / \mathrm{K}, 5 \% / \mathrm{K}$, $7 \% / \mathrm{K}$, and $14 \% / \mathrm{K}$, respectively.

As the analysis in section 4.2 will show, the flexible support influence on the machine dynamics are more pronounced on stiff rotor modes. This single-degree-offreedom model (Fig. 1) shows the scenario in which the compliant support has the highest influence, a rigid rotor. The rotors of actual turbomachines are not rigid and will exhibit some flexibility. Therefore the equivalent parameters of the rigidrotor compliant-support system being discussed would be a theoretical extreme and the actual equivalent parameters would be nearer the rigid support model values than the values shown. Although this analysis is representative of a rigid-rotor single-mode system, it does not capture the influence of the support on a flexible rotor machine operating between the first and second critical speeds.

\subsection{Jeffcott rotor model}

To understand the effect of the compliant support on the dynamic behavior of the Jeffcott rotor model, a representative unbalance response and separation margin 
map are plotted. It should be noted that the trends in Fig. 5-9 are meant to simply show the relative behavior of the system with a varied support stiffness ratio. Two additional comparisons will also be discussed. The first involves the influence of the support stiffness on the first mode compared to the influence on the second mode, and the second is related to variations in the bearing parameters compared to the influence of the support on the damping ratio and damped natural frequency.

\subsubsection{Unbalance response analysis}

The unbalance response at varying support stiffness ratios of the Jeffcott model are plotted in Fig. 5. In agreement with the trends of the single-degree-of-freedom system shown in Tab. 1, the unbalance response plot shows that as the support stiffness ratio is decreased (support becomes more compliant), the amplification factors increase and the critical speeds decrease for both modes.
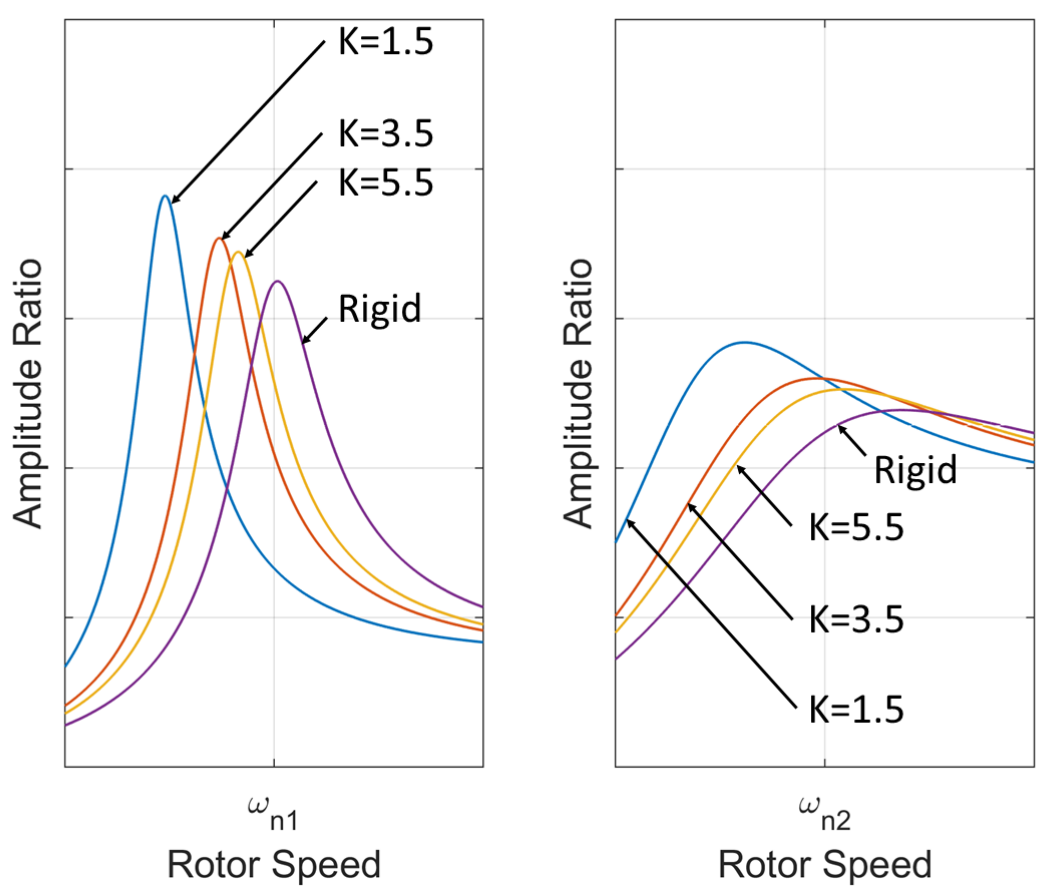

Fig. 5: Unbalance response of Jeffcott rotor model showing the first and second critical speeds with varying support stiffness ratios 


\subsubsection{Damped eigenvalue analysis}

In order to interpret the effects of the compliant support on the separation margins, a separation margin map for the Jeffcott rotor is shown in Fig. 6. Similar to the values in Tab. 1, these amplification factors and frequencies were calculated from the system damped eigenvalues.

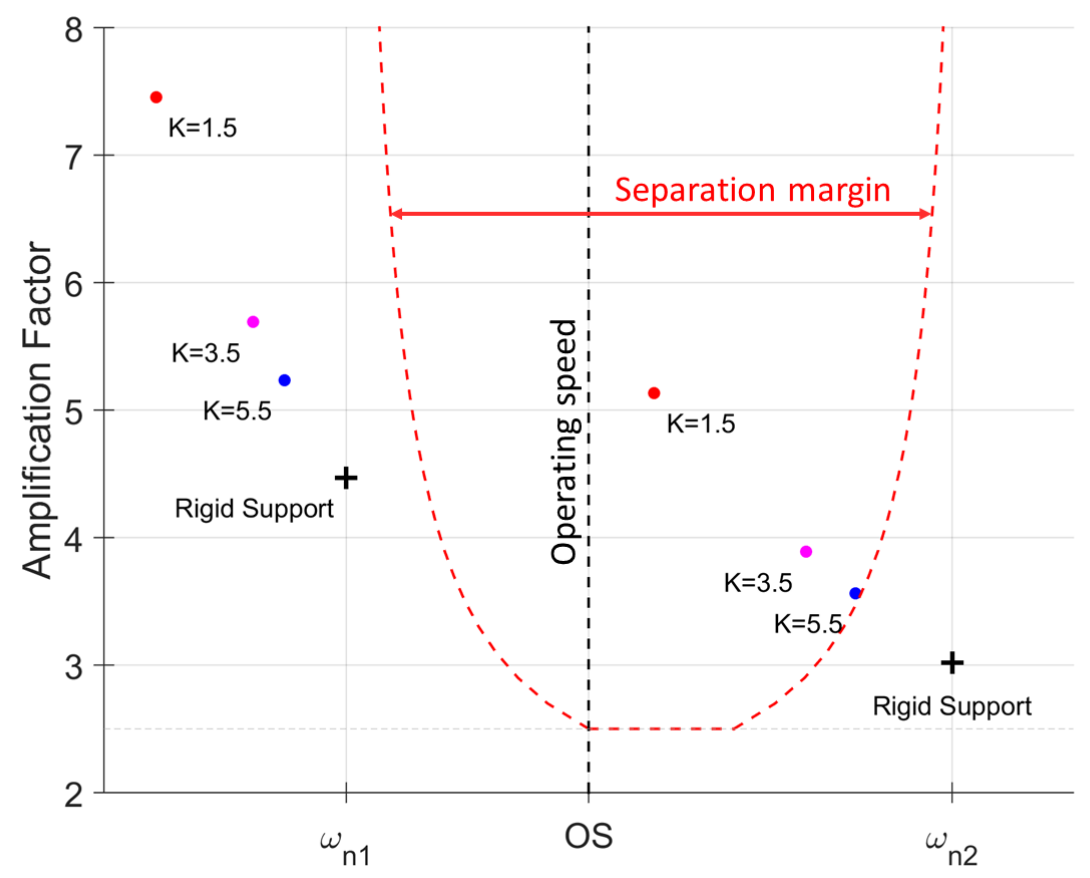

Fig. 6: Separation margin map for Jeffcott rotor model showing increase in amplification factor and decrease in natural frequency for varying support stiffness ratios

The constant operating speed for the example in this map (Fig. 6) was chosen at an arbitrary frequency between the first and second critical speeds. Based on this operating speed choice, the separation margins are plotted. Note that as the amplification factor (plotted on the y-axis) increases, the separation margins widen due to their dependence on the amplification factor. Each pair of points on the map denotes a first and second critical speed positioned at their respective damped natural frequency and amplification factor. In compliance with API standards, all of the critical speeds of the machine should lie outside of this separation margin "bowl." It is 
shown that as the support stiffness ratio decreases (support becomes more compliant) the amplification factors increase and the natural frequencies decrease for both modes.

The separation margin map depicted in Fig. 6 demonstrates the concern that the API support stiffness ratio threshold of 3.5 may not be adequate to protect a machine if its separation margin is near the peak of the rigid support second critical. For example, if the support structure of this system is $5.5 k_{b}(\mathrm{~K}=5.5)$, the engineer may model this machine using a rigid support assumption. It is clearly shown that while the rigid support machine lies outside of the separation margin bowl, the actual machine with the compliant support at $5.5 k_{b}$ violates the separation margin criteria. Actual machinery exhibiting a second mode near the upper bound separation margin may be at risk of the same violation when a rigid support is assumed at the API threshold of $\mathrm{K}=3.5$.

\subsubsection{Rotor stiffness ratio trends}

As mentioned previously, the second mode of the Jeffcott model is more highly influenced by the support stiffness than the first mode. To demonstrate this effect, a plot of the compliant support amplification factor as a percent of the rigid support amplification factor at varying RSR and $\mathrm{K}$ values is shown in Fig. 7. For example, a flexible first and a stiff second rotor mode are highlighted at an RSR of 7.6 and 1.6, respectively. The first system mode line $(\mathrm{RSR}=7.6)$ and the support stiffness ratio line $(K=3.5)$ intersect at a contour level of $5 \%$. This signifies that this first mode will have an amplification factor which is $5 \%$ higher than that of the rigid support first mode. The second mode, known to be stiffer than the first mode, intersects the support stiffness ratio line $(K=3.5)$ at a contour level of $20 \%$. The second mode is shown to have an amplification factor that is $20 \%$ higher than that of the rigid support system. Comparing the first mode influence of $5 \%$ to the second mode influence of $20 \%$ (again, this comparison is strictly relative), it can be seen that stiffer modes 
are more affected by the support stiffness ratio than more flexible modes. In other words, as the rotor becomes stiffer, the support system will carry more modal energy, and its influence will be more pronounced. The same trend is seen with the natural frequencies (Fig. 8). The strong support stiffness effect on the stiff second rotor mode highlights the concern of using the rigid rotor assumption, especially when the second mode is near a separation margin boundary.

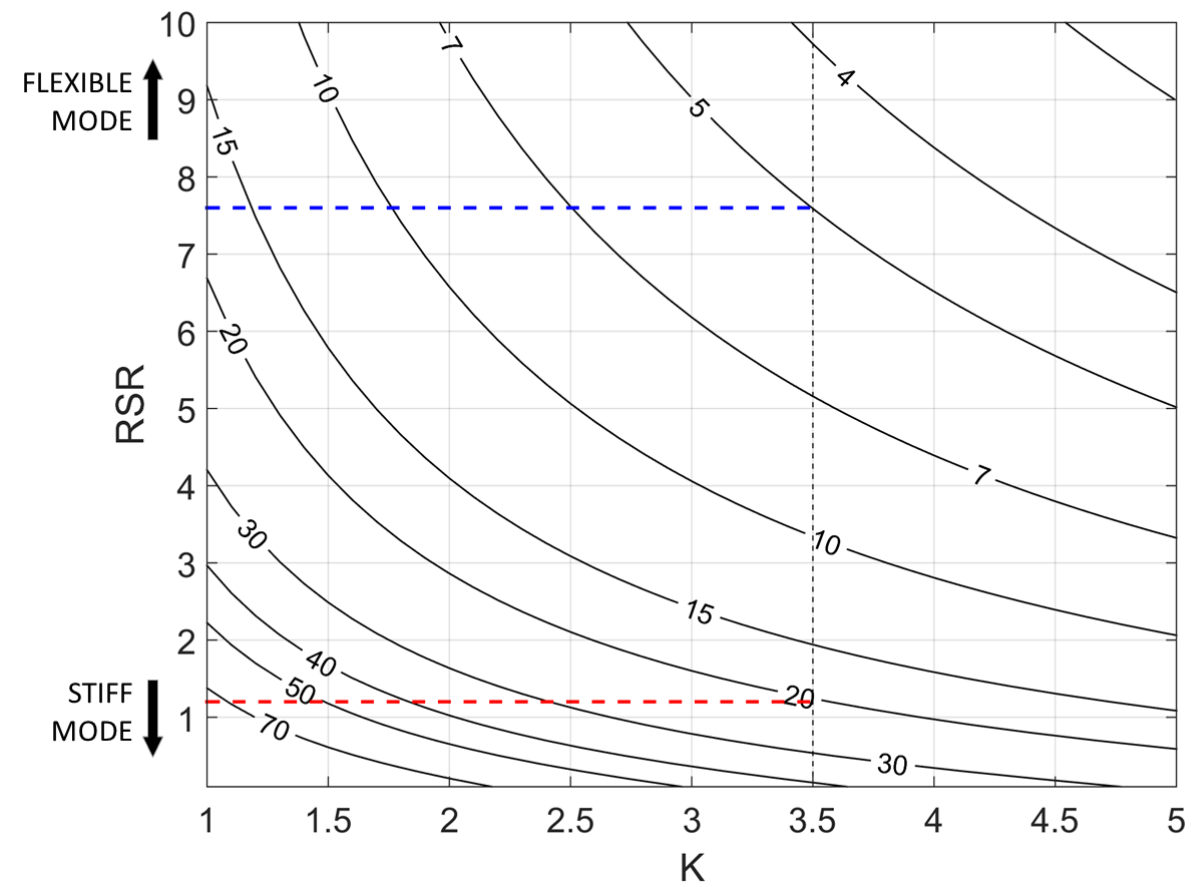

Fig. 7: Percentage increase of rigid rotor amplification factor at various rotor stiffness and support stiffness ratios $\left(\zeta_{b}=0.2\right)$. Horizontal dotted lines suggest a flexible first mode (ex. $\mathrm{RSR}=7.6)$ and a stiff second mode $(\mathrm{ex} . \mathrm{RSR}=1.2)$.

\subsubsection{Bearing parameter variation trends}

In the production of a turbomachine, an unbalance response verification test (URVT) must be performed in order to validate the rotor model used in the design process (Kocur and Cloud 2013). When assuming a rigid support, a common industry practice is to modify the bearing coefficients to make the computational analysis match the URVT data instead of abandoning the rigid support assumption. 


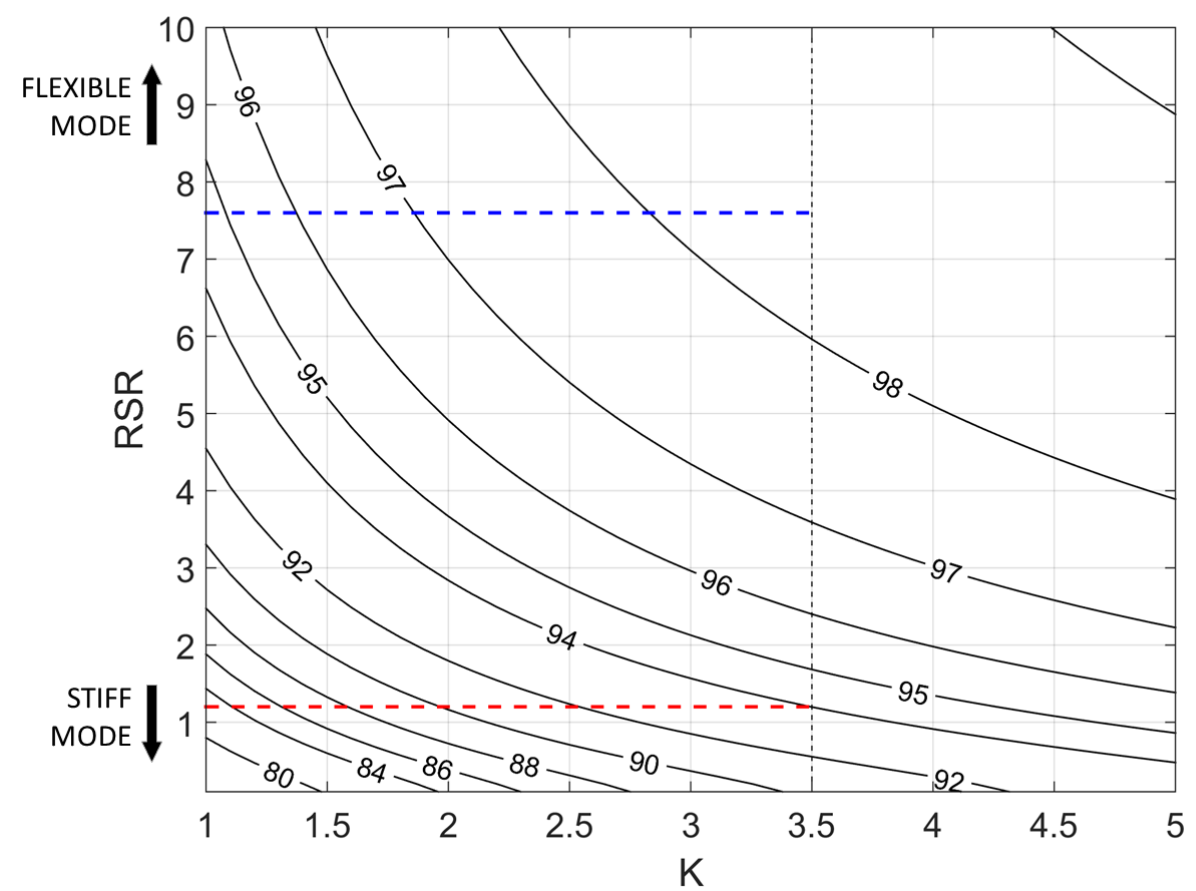

Fig. 8: Percentage of rigid rotor damped natural frequency at various rotor stiffness and support stiffness ratios $\left(\zeta_{b}=0.2\right)$. Horizontal dotted lines suggest a flexible first mode (ex. $\mathrm{RSR}=7.6$ ) and a stiff second mode (ex. $\mathrm{RSR}=1.2$ ).

The bearing parameters are a reasonable component to modify as they are not known precisely and they strongly influence the dynamic behavior of the machine. However, the support structure can also play an important role as discussed in section 4.2.2. This is shown by comparing the damping ratio of a rigid support system, a compliant support system, and a rigid support/altered bearing system at varying rotor stiffness ratios (Fig. 9).

Figure 9 shows the damping ratio of a rigid support/nominal bearing system, a compliant support $(\mathrm{K}=3.5) /$ nominal bearing system, and a rigid support system with a $\pm 10 \%$ bearing coefficient change. The bearing variation shown is the nominal bearing coefficients for stiffness and damping proportionally raised and lowered by $10 \%$ (nominal coefficients being defined by the RSR). The value of $10 \%$ was chosen as it is on the order of the change between the maximum and minimum bearing coefficients used in the case studies in Sec. 5. This figure suggests that for a relatively 


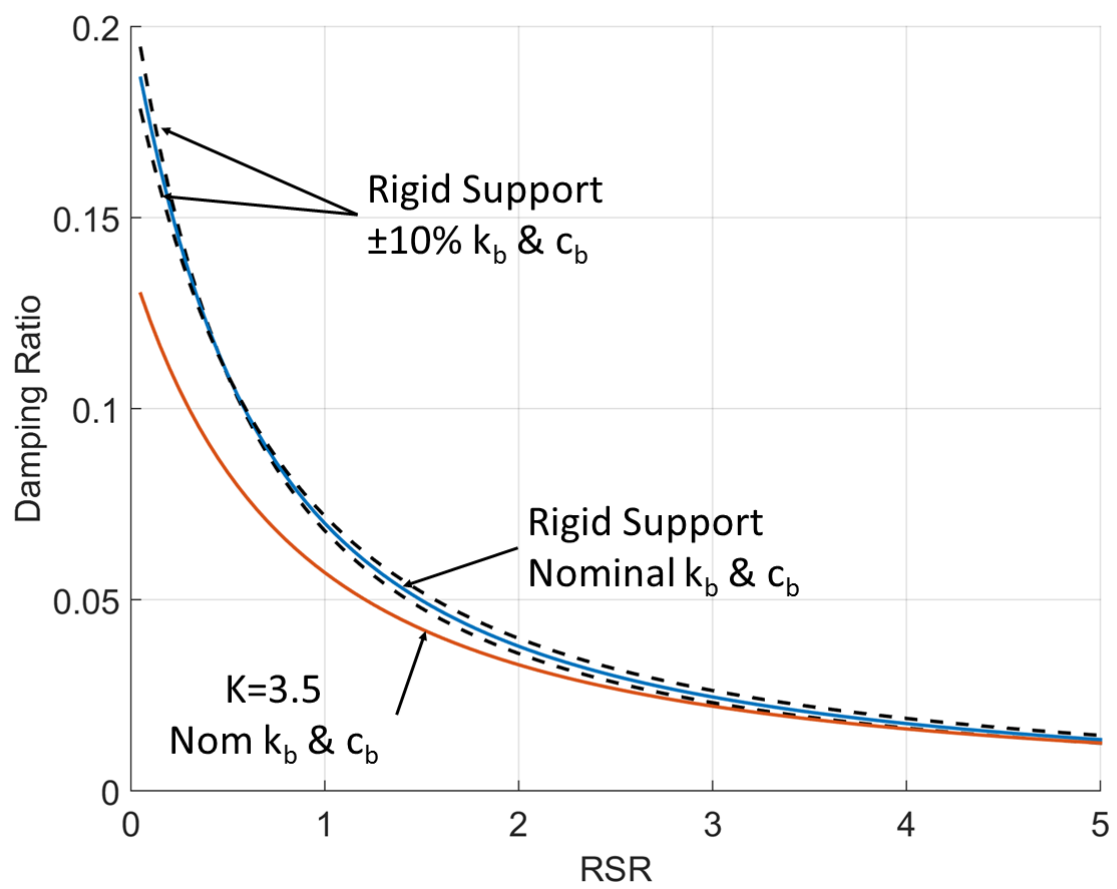

Fig. 9: Damping ratio of rigid support/nominal bearing, compliant support $(\mathrm{K}=3.5) /$ nominal bearing, and rigid support/altered bearing systems for varying rotor stiffness ratios (RSR). $\zeta=.2$

stiff second mode (low RSR), the change in modal damping due to a compliant support (at $\mathrm{K}=3.5$ ) will be significantly higher than the influence of a modest variation in the bearing coefficients (of the order of 10\%). A similar trend is seen in the damped natural frequency (Fig. 10), however, the differences are greater for all values of RSR in Fig. 10.

If a rigid support assumption was made in the modeling of a rotor system, Fig. 9 and Fig. 10 show that the support system should be considered for modification in addition to the bearing coefficients if there is discrepancy between the analytical results and experimental data, particularly near the second critical speed. The significance of this section (4.2.4) is to show that, for example, drastic alterations to the bearing might result in an accurate unbalance response however it might not correctly predict a subsequent stability analysis. When a rigid rotor assumption is used, incorrect modification of the rotor model to match the data of the URVT can produce inac- 
curate results in subsequent rotordynamic analyses which cannot be experimentally confirmed. In this type of scenario, including the support in the analysis should be considered even though the support stiffness may be above the $\mathrm{K}=3.5$ threshold.

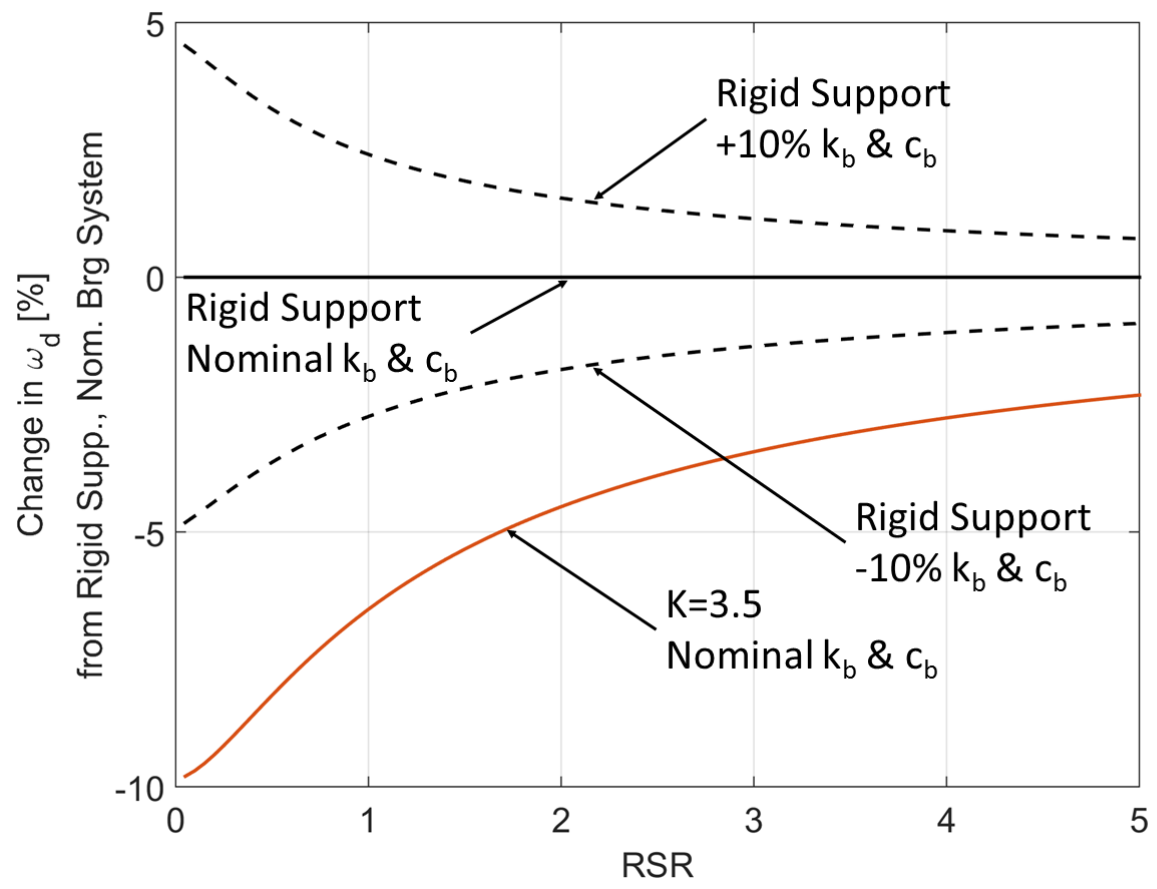

Fig. 10: Percent change in damped natural frequency of rigid support/nominal bearing, compliant support $(\mathrm{K}=3.5) /$ nominal bearing, and rigid support/altered bearing systems for varying rotor stiffness ratios (RSR). $\zeta=.2$

\section{Case study validation}

While the simple one-degree-of-freedom model helped the understanding of the compliant support influence, the Jeffcott model has shown the effect of a compliant support stiffness on the dynamic behavior of a two mode system. This section will validate those trends by analytically investigating the effects of the support stiffness on two industrial machine models. Both case studies will be modeled two ways, using a rigid support and using constant undamped massless symmetric support stiffnesses. The two support configurations will then be compared and the rigid support assump- 
tion at a support stiffness ratio of 3.5 will be discussed.

It should be noted that the aforementioned compliant support configurations are extremely simplified compared to a full-fidelity support model generated from a finite element model or experimental data. The full-fidelity models are those required by the API specification when the support is less than 3.5 times the bearing oil film stiffness.

\subsection{Steam turbine case study}

The first machine is a 17 MW 3-stage steam turbine designed to operate from 4117 to 5085 RPM, between the first and second critical speeds (Fig. 11). The specifications of the steam turbine are shown in Tab. 2. This machine was chosen as a case study because the values supplied by the original equipment manufacturer for the bearing and support stiffnesses resulted in a support stiffness ratio of 3.5. Therefore it was an appropriate model to investigate as it lies on the threshold of the rigid support assumption. The first and second unbalance responses using the minimum, maximum, and nominal bearing coefficients will be analyzed followed by the separation margin map as seen for the Jeffcott rotor in Sec. 4.2.2.

The first and second mode shapes of the steam turbine are shown in Fig. 12. These two mode shapes are analogous to those of the Jeffcott rotor (Fig. 3).

Tab. 2: Specifications for 3-stage steam turbine

\begin{tabular}{lcc}
\hline Operating speed & \multicolumn{2}{c}{$4120-5085 \mathrm{RPM}$} \\
Rotor weight & $2100 \mathrm{~kg}$ & $4640 \mathrm{lbm}$ \\
Bearing stiffness & $280 \mathrm{kN} / \mathrm{mm}$ & $1.6 \mathrm{E} 6 \mathrm{lbf} / \mathrm{in}$ \\
Bearing damping & $315 \mathrm{~N}-\mathrm{s} / \mathrm{mm}$ & $1800 \mathrm{lbf}-\mathrm{s} / \mathrm{in}$ \\
Support stiffness & $981 \mathrm{kN} / \mathrm{mm}$ & $5.6 \mathrm{E} 6 \mathrm{lbf} / \mathrm{in}$ \\
Rigid support eigs (vert) & $3120 \& 6183 \mathrm{RPM}$ \\
\hline
\end{tabular}




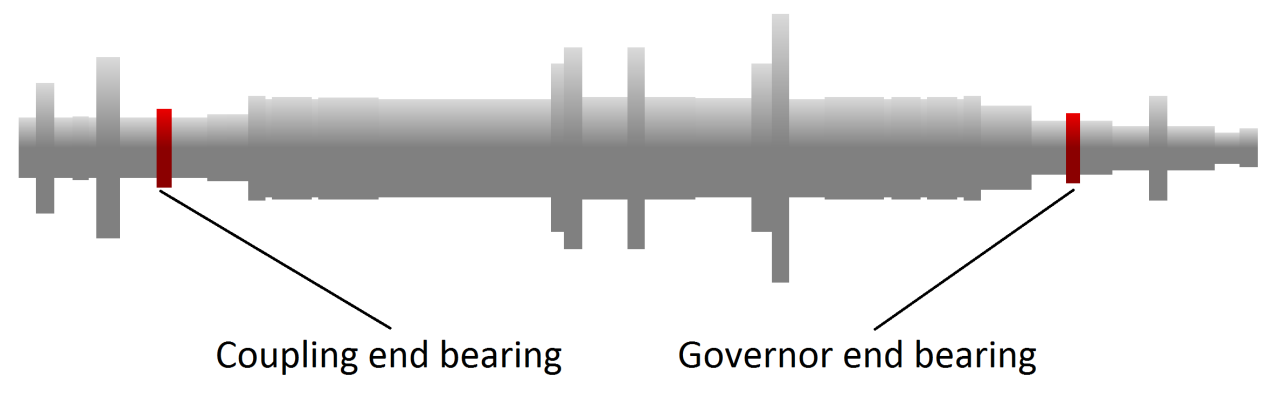

Fig. 11: Schematic of 3-stage steam turbine

\subsubsection{First mode unbalance response}

To reproduce the trends seen by the Jeffcott model, the first and second mode unbalance responses of the steam turbine were first examined and these are shown in Fig. 13 and Fig. 14, respectively. Referring to the results in Fig. 13, the unbalance response of the first mode with a compliant support $(K=3.5)$ shows little change in the amplification factor and only a slight decrease in the first critical speed compared to the rigid support system. This is expected as the first mode is not as highly influenced by the support as the second mode. Note the offset dotted response lines; these lines depict the responses of the system based on the maximum and minimum bearing coefficients. The maximum and minimum bearing coefficients are generated from the extremes of the design clearances of the bearings as well as including the extreme values of the inlet lubricant temperatures API $6842^{\text {nd }}$ Edition 2005. Since this machine operates above the first critical speed, modeling this system with a rigid support is actually a conservative assumption from a separation margin standpoint. The rigid support model will result in a first critical speed nearer the operating range than the compliant support model, ultimately resulting in an observed machine critical at a safer distance from the operating speed. This conservative trend is only seen on the first mode since it is below the operating speed. It is shown that neither of 


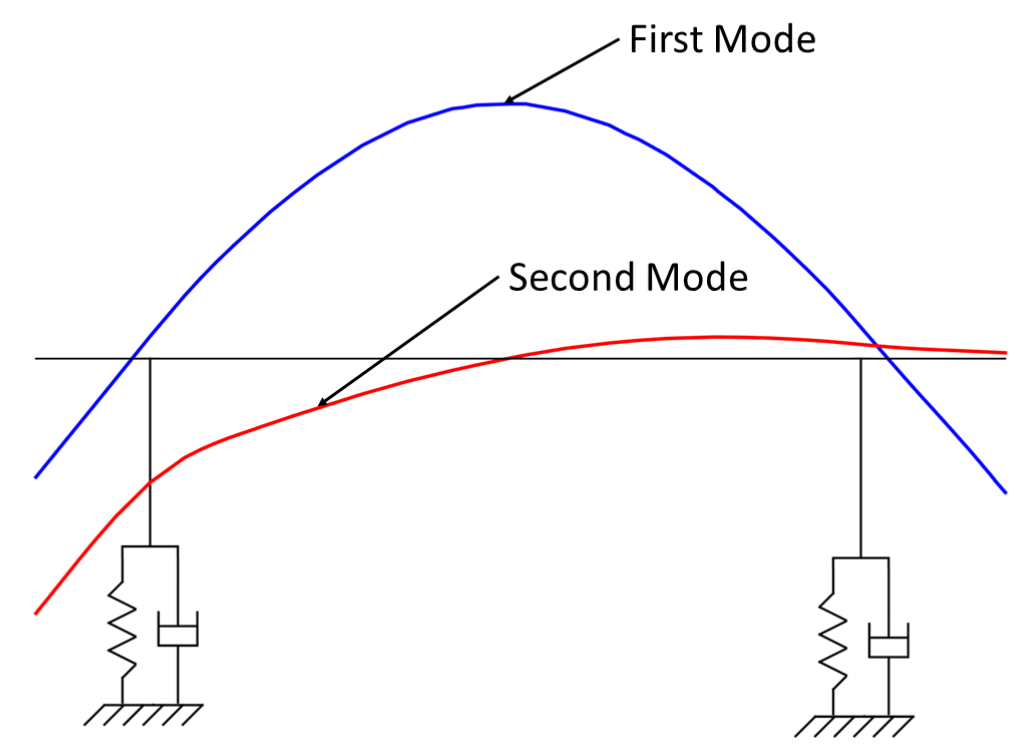

Fig. 12: First and second vertical modes of steam turbine case study

the two modeling approaches will result in a first critical speed inside the separation margins of the machine.

The rigid support assumption is generally conservative for any machine operating above the first critical speed given that the change in amplification factor is small between the rigid and compliant support models. However, if the amplification factor for the compliant support model is high enough to increase the separation margin to a frequency above that of the rigid support model, the assumption will not be conservative. In this scenario, the API support stiffness ratio threshold of 3.5 allows for a rigid support assumption which will only make the machine safer from a separation margin standpoint due to the small change in amplification factor of the first mode.

\subsubsection{Second mode unbalance response}

The second critical speed unbalance plot (Fig. 14) shows a significant change between the rigid and compliant $(\mathrm{K}=3.5)$ support systems. The vibration amplitudes 


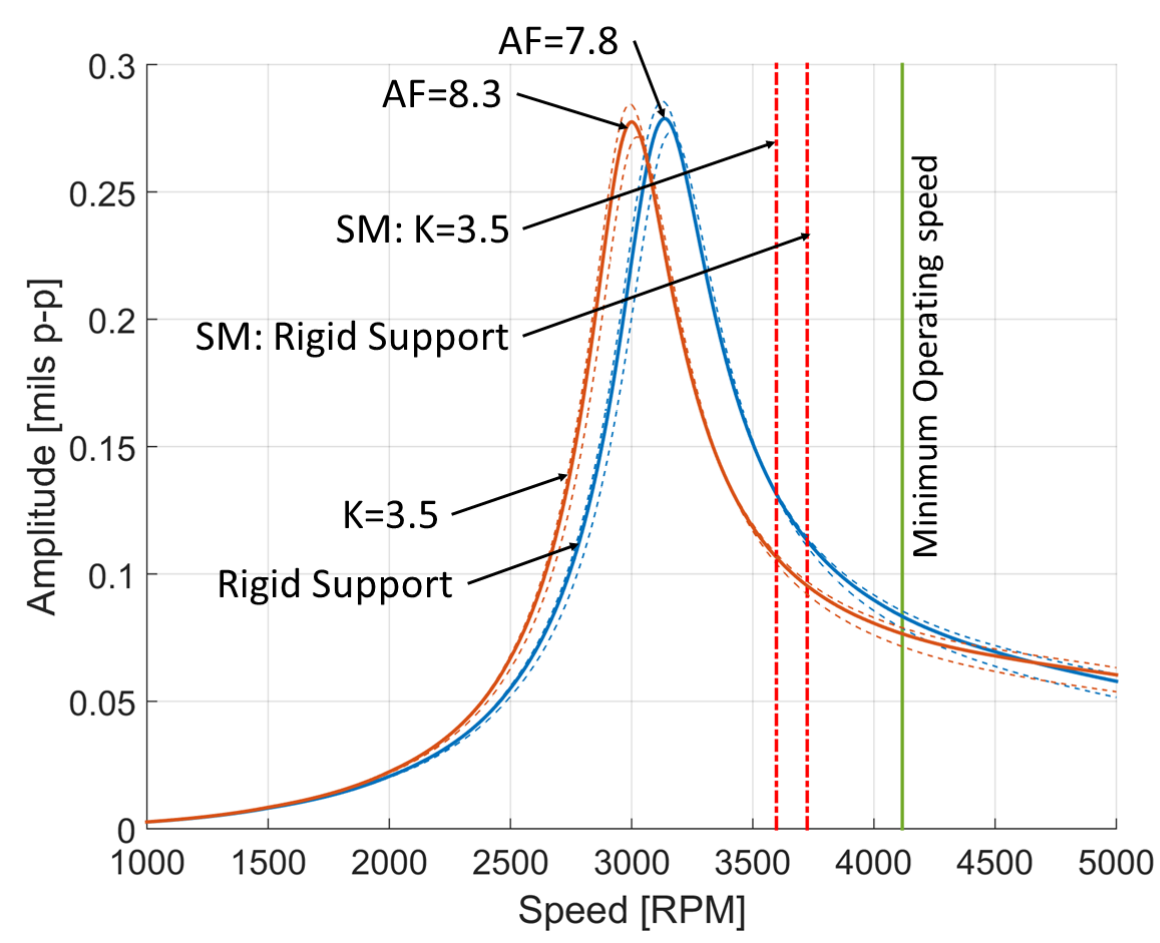

Fig. 13: First mode relative unbalance response of rigid and compliant $(\mathrm{K}=3.5)$ support systems. Offset dotted lines show responses using minimum and maximum bearing coefficients. Vertical response at coupling end bearing. Separation margins show how far away from the critical speed the machine is required to operate.

and amplification factors of the compliant support system have increased by approximately $27 \%$ and the critical speeds have decreased by approximately 450 RPM. These values are organized in Tab. 3. The required separation margins for both support system responses are drawn and it is shown that the separation margin for the compliant support $(\mathrm{K}=3.5)$ response falls inside the operating speed range of the machine. Per API standards, this support stiffness ratio at the threshold of 3.5 may be modeled with a rigid support. While the rigid support system is acceptable by the API standards, the compliant support at $\mathrm{K}=3.5$ is clearly in violation. In this scenario, the API support threshold specification does not fully protect this machine under the rigid support assumption criteria. This second mode unbalance response plot demonstrates the importance of including the support structures for machines with operating speeds near the rigid support second critical speed. 


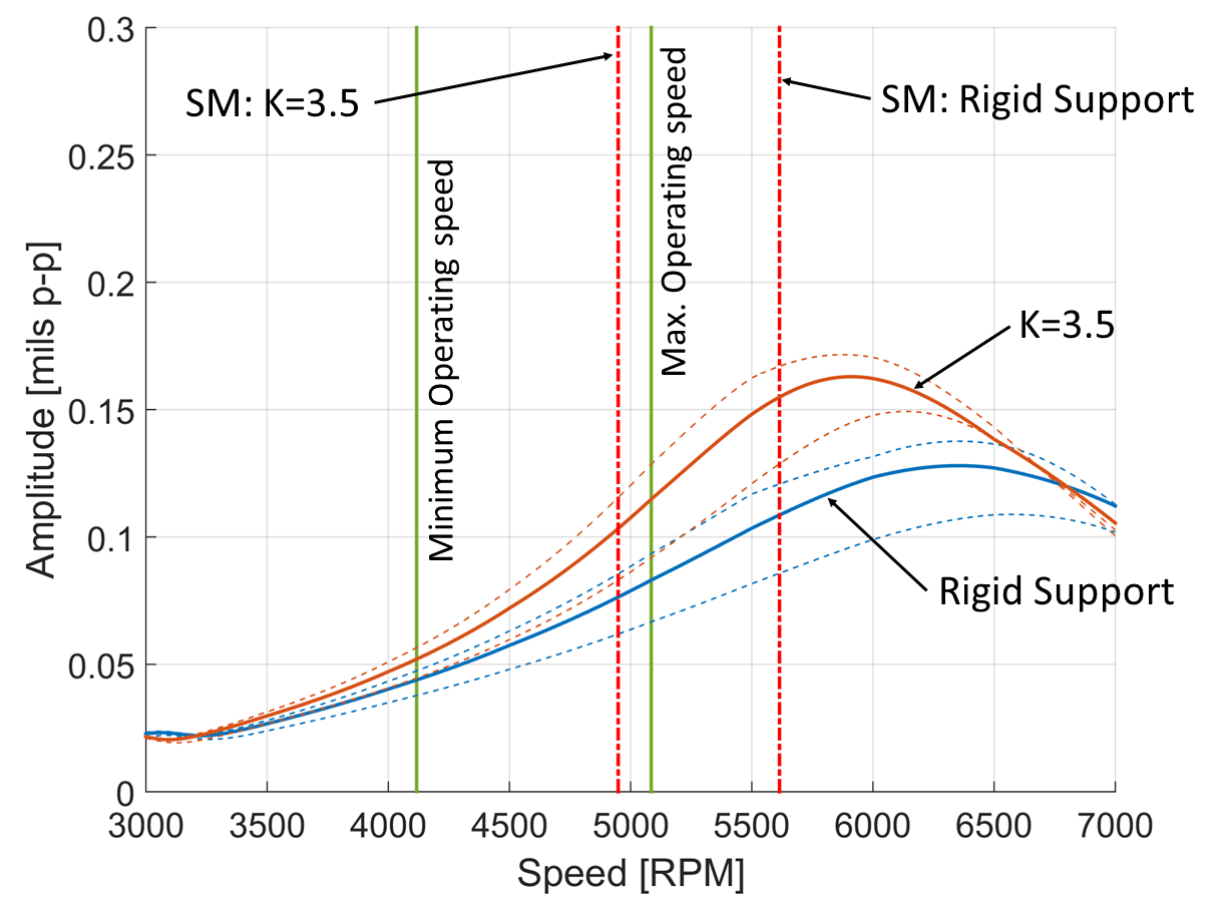

Fig. 14: Second mode relative unbalance response of rigid and compliant $(\mathrm{K}=3.5)$ support systems. Offset dotted lines show responses using minimum and maximum bearing coefficients. Vertical response at coupling end bearing.

\subsubsection{Bearing parameter variation}

As discussed in section 4.2.4, for stiff rotor modes with a support stiffness ratio of 3.5, the dynamic influence due to a slight change in bearing coefficients is dominated by the change induced by the flexible support. Tab. 3 shows that the max and min bearing coefficients only change the amplification factor of the second mode by about $7 \%$ of the rigid support amplification factor, while the compliant support influence changes it by as much as $29 \%$. The natural frequencies follow a similar trend; the max and min bearing coefficients only change the critical speed by approximately 215 RPM whereas the compliant support changes it by as much as 440 RPM. The unbalance response shows that the max and min bearing coefficients have little effect on the dynamic behavior, especially for the first mode. The changes due to the extremes of the bearing coefficients compared to the influence of the compliant support show 
Tab. 3: Steam turbine 2nd mode amplification factors and critical speeds at varying support and bearing parameters. Calculated from unbalance response (Fig. 14). $($ Nom=Nominal $)$

\begin{tabular}{lccccc}
\hline Support & Bearing & RPM & AF & \%Rig. AF & $\Delta$ Rig. RPM \\
\hline Rigid & Min & 6565 & 2.76 & $97 \%$ & 215 \\
Rigid & Nom & 6350 & 2.86 & $100 \%$ & 0 \\
Rigid & Max & 6350 & 2.66 & $93 \%$ & 0 \\
K=3.5 & Min & 6135 & 3.70 & $129 \%$ & -215 \\
K=3.5 & Nom & 5910 & 3.60 & $126 \%$ & -440 \\
K=3.5 & Max & 5875 & 3.34 & $117 \%$ & -475 \\
\hline
\end{tabular}

that when a rigid support assumption is used, alterations to a rotor model to match experimental data should include the support structure in addition to the bearings.

\subsubsection{Damped eigenvalue analysis}

The separation margin map, as discussed in section 4.2.2 has also been created for the steam turbine case study (Fig. 15). The map shows the same trend seen from the Jeffcott rotor model; however, the case study shows two forward modes and two backward modes as opposed to the two single modes of the Jeffcott rotor. The Jeffcott rotor used in the analysis in section 4 omits gyroscopic and cross-coupling effects which couple the $\mathrm{x}$ and $\mathrm{y}$-axes; because of this, the rotor may be decoupled and a single axis of freedom may be studied. In the case study models, the two axes are coupled, due to gyroscopic effects and bearing cross-coupling, and therefore result in eigenvalues in each axis and modes of forward and backward precession (E. Gunter and W. Chen 2000). Figure 15 shows that as the support stiffness ratio is decreased, the first and second natural frequencies decrease and the amplification factors increase. Note the significant change seen on the second mode compared to the first mode.

The separation margin map shows that the second forward mode at a support stiffness ratio of 3.5 is directly on the upper separation margin boundary. Keep in 


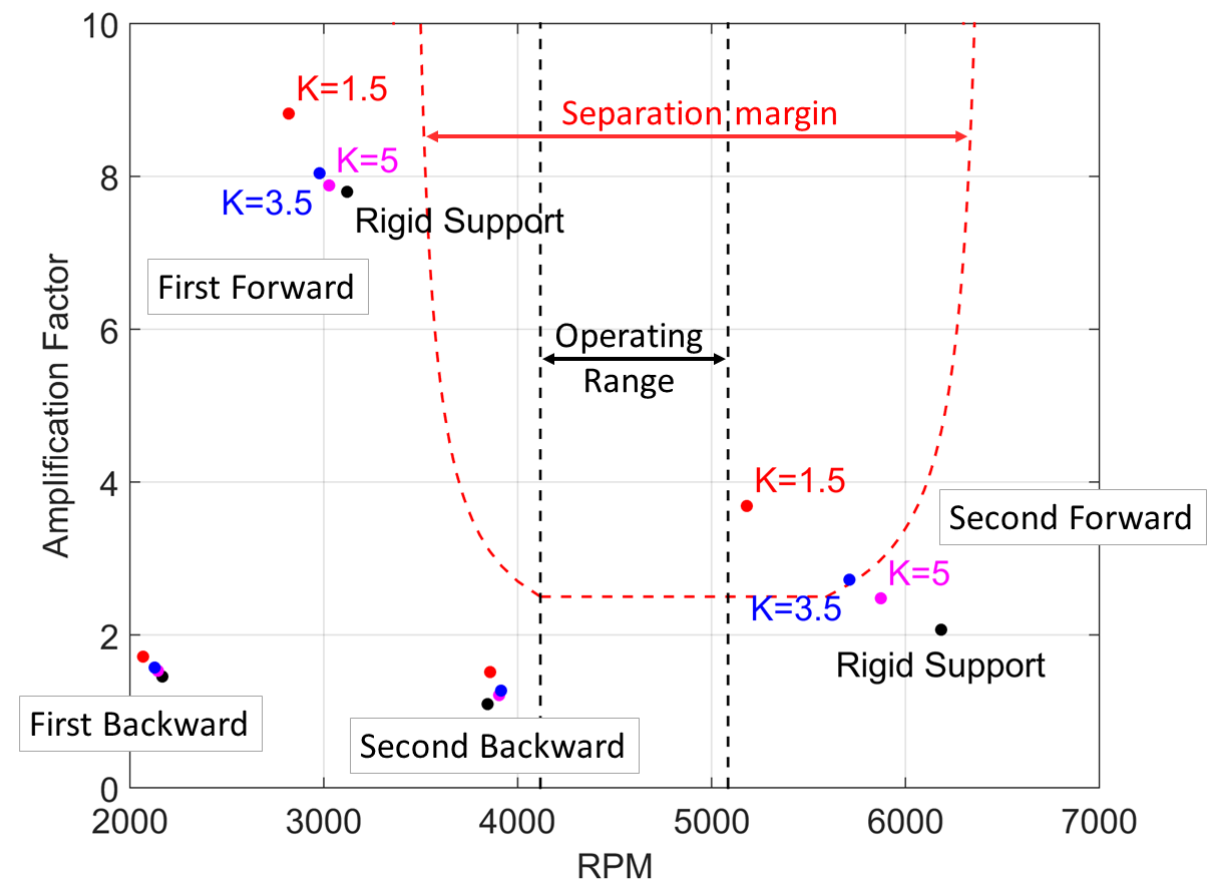

Fig. 15: Separation margin map for steam turbine. Two sets of well-damped backwards modes (lower left corner) are omitted from the analysis.

mind that the amplification factors and damped natural frequencies of these modes are calculated using the damped eigenvalues of the system. The separation margins used in API standards are those calculated from the unbalance response, as the amplification factor can easily be calculated from experimental data (API $6842^{\text {nd }}$ Edition 2005). Recall that the separation margin from the second critical shown in section 5.1.2 was well inside the operating speed range. Although the eigenvaluederived second forward mode at a support stiffness ratio of $\mathrm{K}=3.5$ (Fig. 15) lies directly on the boundary of the separation margin bowl, the trend still suggests that this is a mode of concern. As the support compliance increases, the mode is trending in an unfavorable direction, to a higher amplification factor and closer to the operating speed range. 


\subsection{Centrifugal compressor case study}

The second case study is a centrifugal compressor designed to operate from 4800 to 6400 RPM, also between the first and second critical speeds (Fig. 16). The specifications of the compressor are shown in Tab. 4. This case study will further demonstrate the strong influence of the compliant support on the second critical speed by analyzing the damped eigenvalues of the system. The unbalance responses of this machine will not be presented as the first mode shows a similar behavior as the previous case study, and the provided bearing coefficients were not calculated at speeds high enough to accurately calculate the second mode amplification factors.

The first and second mode shapes of the compressor are shown in Fig. 17. These two mode shapes are analogous to those of the Jeffcott rotor (Fig. 3).

\subsubsection{Damped eigenvalue analysis}

The separation margin map was generated for the centrifugal compressor and is shown in Fig. 18. Again, the second forward mode is strongly influenced by the compliant support while the more flexible first forward mode shows less influence. At a support stiffness ratio of 3.5, the second forward mode is on the boundary of the separation margin and results in an API separation margin violation. Also note that this machine is running with a well-damped second backwards mode inside the operating speed range. This mode should be taken into consideration, especially at low support stiffness ratios. As the support stiffness ratio is decreased, the well-damped second backward mode is becoming more prominent and could possibly generate a critical speed in the unbalance response. This case study is a good example of a machine that is designed to safely operate with a well-damped critical speed in the operating range. Although this particular second backward mode is not in violation of any API separation margin criteria, it demonstrates the potential risk of designing a machine to run on a well-damped critical while using a rigid support assumption. This separa- 


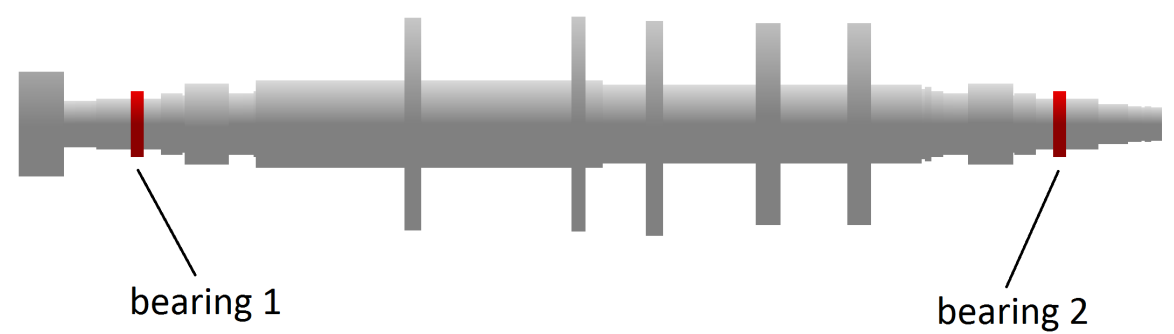

Fig. 16: Schematic of centrifugal compressor

Tab. 4: Specifications for centrifugal compressor

\begin{tabular}{lcc}
\hline Operating speed & \multicolumn{2}{c}{$4800-6400 \mathrm{RPM}$} \\
Rotor weight & $1620 \mathrm{~kg}$ & $3576 \mathrm{lbm}$ \\
Bearing stiffness & $210 \mathrm{kN} / \mathrm{mm}$ & $1.2 \mathrm{E} 6 \mathrm{lbf} / \mathrm{in}$ \\
Bearing damping & $203 \mathrm{~N}-\mathrm{s} / \mathrm{mm}$ & $1160 \mathrm{lbf}-\mathrm{s} / \mathrm{in}$ \\
Rigid support eigs (vert) & $2475 \& 7292 \mathrm{RPM}$ \\
\hline
\end{tabular}

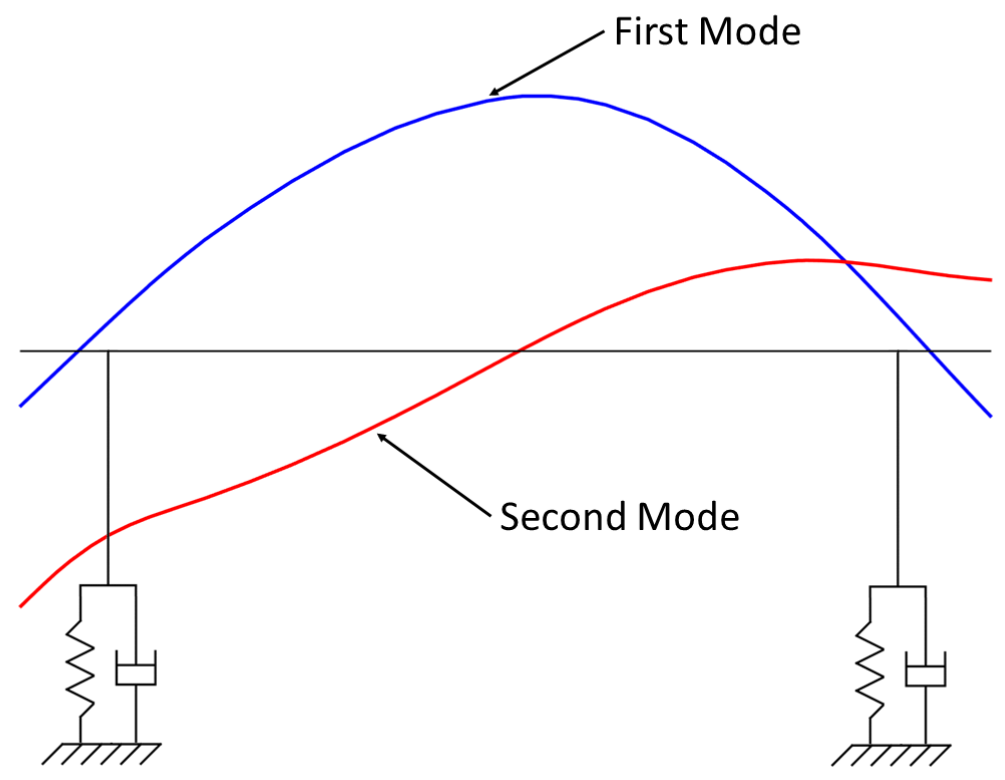

Fig. 17: First and second vertical modes of centrifugal compressor case study 


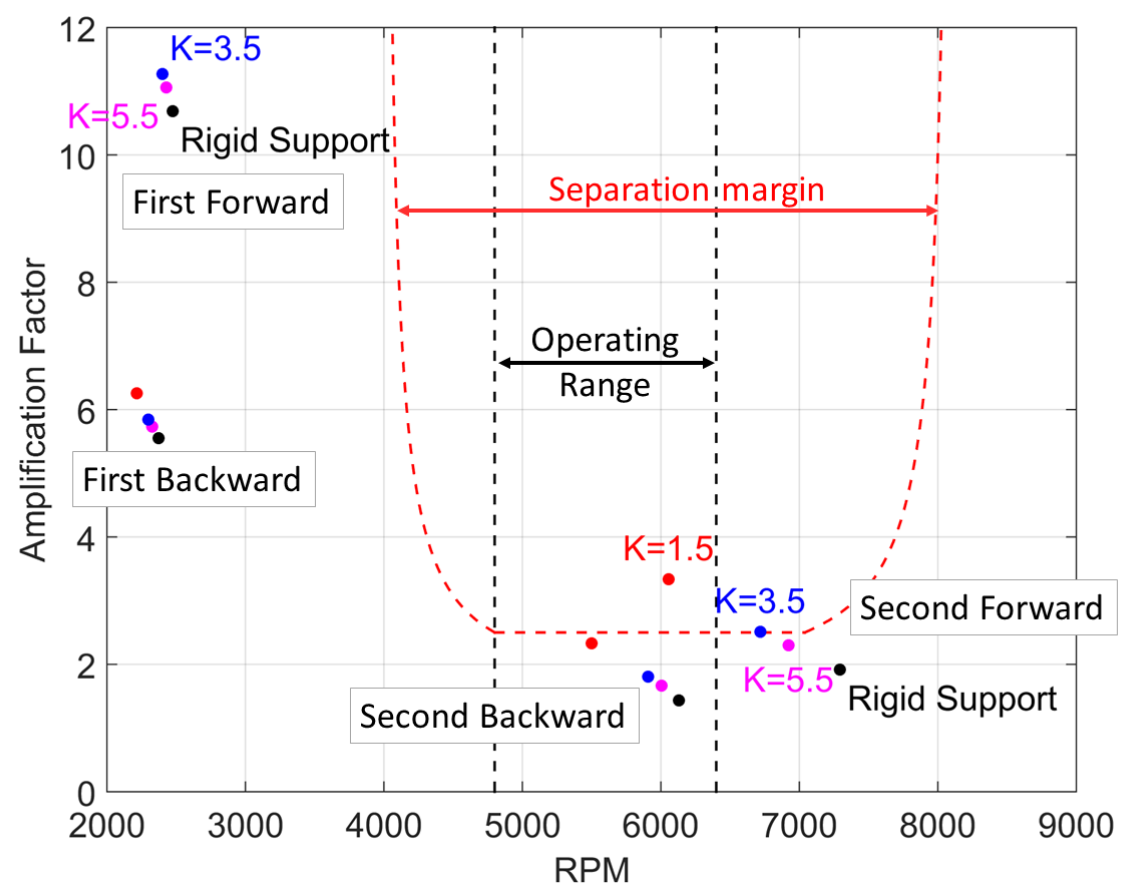

Fig. 18: Separation margin map for centrifugal compressor

tion margin map demonstrates that the second forward and also the second backward modes may not be well-suited for a rigid support assumption at a support stiffness ratio of 3.5 .

\section{Discussion}

\subsection{Questions of purpose}

This investigation has shown the influence of the support stiffness on the unbalance response and damped eigenvalues in very simple rotor models. The trends from the analysis were then validated using computational case studies associated with two industrial machines. The five questions proposed in the Introduction can now be answered and are listed below.

1. What is the basis for the support stiffness ratio of 3.5 as a minimum? 
The exact analytical value of 3.5 could not be derived from the simplified models used in this analysis. The single-degree-of-freedom model (Sec. 4.1) shows that at a ratio of $K=3.5$, the equivalent natural frequency, stiffness, and damping values are asymptotically approaching those of the rigid support system (Fig. 4.1) at a rate of less than $3 \% / \mathrm{K}, 5 \% / \mathrm{K}$, and $7 \% / \mathrm{K}$, respectively. The separation margin maps for the two case studies show that the damped natural frequencies, with a stiffness ratio of 3.5, are very near the separation margin boundaries. Judging from these case studies, one might conclude that this number was derived from experience in analyzing a number of real machines.

2. What are the practical implications of the 3.5 support stiffness ratio?

As the support stiffness ratio decreases, the system natural frequencies and the equivalent damping decrease. The decrease in effective damping results in a higher amplification factor for the given modes. With a relatively stiff second rotor mode, there is concern that the support stiffness effect may be significant enough to move the second critical speed into the separation margins or even the operating speed range.

3. What are some characteristics of a dynamic system where a support stiffness ratio of 3.5 becomes a relevant concern?

Dynamic systems with high bearing stiffness may be at higher risk as they will have lower support stiffness ratios for a given support stiffness. Also, systems with stiff second rotor modes will be more influenced by the support. 
4. What classes or types of machinery have these dynamic properties?

i) Machines with heavy rotors producing high bearing stiffnesses will have support stiffness ratios approaching 3.5.

ii) If a machine is designed to operate on a well-damped critical speed, this critical may lose its effective damping and become a critical speed of concern in the operating speed range.

iii) Machines with compliant supports operating at a frequency near a highly amplified second mode will have a high risk of separation margin violation under the rigid support assumption.

5. Are there any better or additional guidelines that could be used to reduce or eliminate the above concerns?

This analysis has shown that the influence of the support on the dynamic behavior of a supercritical turbomachine cannot be captured by a support stiffness value alone. In order to better eliminate the above concerns, an addendum to the current API support stiffness ratio specification, presented here, is to require a simplified support model for machines with a support stiffness ratio greater than 3.5. A detailed explanation of the suggested addendum is found in section 6.4 .

\subsection{Post-design support considerations}

This investigation has focused mainly on the influence of the support stiffness ratio on the new design of turbomachinery. Another scenario that should be con- 
sidered is the degradation or variation of support characteristics after the machine has been in service. Over time the rigidity of supporting structures can degrade and cause a change in the dynamics of the machine. As machinery is disassembled and reassembled over the course of its life, the fits of housings and alignments can loosen and skew, resulting in altered dynamic properties. A post-installation modification to a structure can cause directional stiffening and a shift in the system behavior. As shown in section 4.2.4, uncertainty in the support stiffness may produce larger changes in the damping ratio and damped natural frequencies than a $10 \%$ change in bearing coefficients. The change over time should be considered when analyzing a machine that has been in service as the dynamics can be different from the as-built state.

\subsection{Support influence on subcritical machines}

Machines designed to operate below the first critical speed may also exhibit separation margin encroachment. Because the first mode is more flexible than the second, the compliant support will not influence the natural frequency and effective damping as much as the supercritical scenarios analyzed in section 4.2. The concern of separation margin encroachment is dependent upon the bandwidth between the separation margin and the rigid support critical speed. The analysis has shown that a subcritical machine may safely operate closer to a rigid support critical speed than a supercritical machine due to the reduced influence of the compliant support at the ratio of $\mathrm{K}=3.5$.

\subsection{Suggestion for API specification}

In order to eliminate the concern of separation margin encroachment, an addition to the API support stiffness specification is suggested as follows:

"The term "support" includes the foundation or support structure, the 
base, the machine frame and the bearing housing as appropriate.

i) For machines whose bearing support system stiffness values are less than or equal to 3.5 times the bearing oil film stiffness values, support stiffness values derived from modal testing or calculated frequency dependent support stiffness and damping values (impedances) shall be used.

ii) For machines whose bearing support system stiffness values are greater than 3.5 times the bearing oil film stiffness values, a massless support with stiffness equal to that of the estimated support stiffness shall be used."

In this addendum, the "estimated support stiffness" should be the same stiffness value used to determine if the support stiffness ratio is above or below 3.5 in 6.4.i. The simplified massless support model mentioned above is a compromise between a rigid support assumption and a high-fidelity computational model of the support. Including a support stiffness between each bearing and ground is a quick addition to any rotordynamic model using modern software. The inclusion of the support stiffness will predict the second critical speed behavior more accurately and reduce the risk of separation margin encroachment.

\section{Conclusion}

The current API support stiffness ratio specification is intended to allow engineers to safely assume a rigid support if the machine satisfies the stiffness ratio criteria. This analysis has shown that, for a super critical machine, the rigid support assumption results in a generally conservative first mode response but poses a threat for a machine operating just below the second critical speed. As machines are designed to have increased power density, more machines will be running near the second critical. 
This investigation has demonstrated that the API support stiffness ratio threshold, although convenient in its simplicity, can result in potential limitations when the second critical speed of a machine operating on a rigid support is close to the separation margin boundary.

Many authors, such as Kirk and Gunter (1972), Nicholas and Barrett (1986), Nicholas (1989), Vázquez, et al (2001), among others, have studied the support effect on the dynamic behavior of rotating machinery but this paper is the first to investigate the effect by comparing a compliant support system to a rigid support system in accordance with the API support stiffness threshold. The analysis is intended to caution the industry by demonstrating the risk involved with blindly assuming a rigid support for machines that meet the current API support stiffness threshold. The suggested addendum to the current API specification (Sec. 6.4) will eliminate the concern of separation margin encroachment due to a rigid support assumption. The addition is simple, can be performed quickly, and will help ensure a well-behaved machine. 


\section{BIBLIOGRAPHY}

API $6114^{\text {th }}$ Edition (1997). General-Purpose Steam Turbines for Petroleum, Chemical, and Gas Industry Services. Washington, DC: API Publishing Services.

API $6126^{\text {th }}$ Edition (2005). Petroleum, Petrochemical and Natural Gas IndustriedSteam Turbines- Special Purpose Applications. Washington, DC: API Publishing Services.

API $6177^{\text {th }}$ Edition (2002). Axial and Centrifugal Compressors and Expanderscompressors for Petroleum, Chemical and Gas Industry Services. Washington, DC: API Publishing Services.

API $6724^{\text {th }}$ Edition (2004). Packaged, Integrally Geared Centrifugal Air Compressors for Petroleum, Chemical, and Gas Industry Services. Washington, DC: API Publishing Services.

API $6841^{\text {st }}$ Edition (1996). API Standard Paragraphs Rotordynamic Tutorial: Lateral Critical Speeds, Unbalance Responses, Stability, Train Torsionals and Rotor Balancing. Washington, DC: API Publishing Services.

API $6842^{\text {nd }}$ Edition (2005). API Standard Paragraphs Rotordynamic Tutorial: Lateral Critical Speeds, Unbalance Responses, Stability, Train Torsionals and Rotor Balancing. Washington, DC: API Publishing Services.

Barrett, LE, EJ Gunter, and PE Allaire (1978). "Optimum bearing and support damping for unbalance response and stability of rotating machinery". In: Journal of Engineering for Power 100.1, pp. 89-94. 
Birchfield, Neal, Kumar Vikram Singh, and Sumit Singhal (2013). "Dynamical structural modification for rotordynamic application". In: ASME 2013 International Design Engineering Technical Conferences and Computers and Information in Engineering Conference. American Society of Mechanical Engineers, V008T13A056V008T13A056.

Caruso, William J, Bruce E Gans, and William G Catlow (1982). "Application of recent rotor dynamics developments to mechanical drive turbines". In: Proceedings of the Eleventh Turbomachinery Symposium.

Chen, Wen Jeng and Edgar J Gunter (2010). Dynamics of rotor-bearing systems. Trafford publishing, Canada.

Dikmen, Emre et al. (2009). "A Flexible Rotor on Flexible Supports: Modeling and Experiments". In: ASME 2009 International Mechanical Engineering Congress and Exposition. American Society of Mechanical Engineers, pp. 51-56.

Gunter, Edgar J (1983). Rotor-bearing stability. Vibration Institute.

Gunter, Edgar J and P De Choudhury (1969). Rigid rotor dynamics. Vol. 1391. National Aeronautics, Space Administration; for sale by the Clearinghouse for Federal Scientific, and Technical Information, Springfield, Va.

Gunter, EJ and WJ Chen (2000). "DyRoBeS-Dynamics of rotor bearing systems user's manual". In: Rodyn Vibration Analysis Inc, Charlottesville, VA.

Kang, Y et al. (2000). "An investigation in stiffness effects on dynamics of rotorbearing-foundation systems". In: Journal of Sound and Vibration 231.2, pp. 343374.

Kimball Jr, AL (1925). "LXXVI. Internal friction as a cause of shaft whirling". In: The London, Edinburgh, and Dublin Philosophical Magazine and Journal of Science 49.292, pp. $724-727$. 
Kirk, RG and De Choudhury (1975). "The effect of support flexibility on the stability of rotors mounted in plain cylindrical journal bearings". In: Dynamics of rotors, pp. $244-298$.

Kirk, Robley Gordon and Edgar J Gunter (1972). "The effect of support flexibility and damping on the synchronous response of a single-mass flexible rotor". In: Journal of Engineering for Industry 94.1, pp. 221-232.

Kocur, John A and C Hunter Cloud (2013). "Shop Rotordynamic Testing- Options, Objectives, Benefits and Practices". In: Proceedings of the Forty-Second Turbomachinery Symposium.

Lund, Jorgen W (1965). "The stability of an elastic rotor in journal bearings with flexible, damped supports". In: Journal of Applied Mechanics 32.4, pp. 911-920.

MATLAB (2015). version 8.5.0 (R2015a). Natick, Massachusetts: The MathWorks Inc.

Nicholas, JC and LE Barrett (1986). "The effect of bearing support flexibility on critical speed prediction". In: ASLE transactions 29.3, pp. 329-338.

Nicholas, John C (1989). "Operating Turbomachinery On or Near the Second Critical Speed in Accordance with API Specifications". In: Proceedings of the Eighteenth Turbomachinery Symposium, pp. 47-54.

Nicholas, John C, John K Whalen, and Sean D Franklin (1986). "Improving critical speed calculations using flexible bearing support FRF compliance data". In: Proceedings of the 15th Turbomachinery Symposium, pp. 69-78.

Rao, S.S. (2011). Mechanical Vibrations, Fifth Edition. Upper Saddle River, NJ: Pearson Education. 
Vázquez, José A and Lloyd E Barrett (1998). "Representing flexible supports by polynomial transfer functions". In: ASME 1998 International Gas Turbine and Aeroengine Congress and Exhibition. American Society of Mechanical Engineers, V005T14A008-V005T14A008.

Vázquez, José A, Lloyd E Barrett, and Ronald D Flack (2001a). "A flexible rotor on flexible bearing supports: stability and unbalance response". In: Journal of Vibration and Acoustics 123.2, pp. 137-144.

- (2001b). "Including the effects of flexible bearing supports in rotating machinery". In: International Journal of Rotating Machinery 7.4, pp. 223-236. 
APPENDIX 


\section{Appendix A: Amplification Factor and Separation Margin}

The following sections will define and explain amplification factors (AF) and separation margins (SM). Figure 19 is an example of an unbalance response plot. The $\mathrm{x}$-axis is running speed and the $\mathrm{y}$-axis is amplitude ratio. Amplitude ratio, not to be confused with amplification factor, is a non-dimensional parameter which quantifies the ratio of the output vibration magnitude to the input unbalance eccentricity. The equations for the unbalance force and amplitude ratio are shown in Eqn. 5 and 6 respectively.

$$
\begin{gathered}
F_{\text {unbalance }}=\text { mew }^{2} \\
\text { Amplitude ratio }=\frac{X}{e}
\end{gathered}
$$

Where $m$ is the unbalance mass, $e$ is the unbalance eccentricity, $\omega$ is the running speed, and $X$ is the resulting vibration magnitude. A high amplitude ratio denotes a high vibration amplitude relative to a given amount of unbalance.

\section{Amplification factor}

The two peaks shown in Fig. 19 denote a first and second critical speed. Each of these critical speeds will have an amplification factor. The AF is a non-dimensional value which describes how sensitive the vibration amplitude of a certain mode is to a change in excitation frequency. In the case of an unbalance response, this excitation frequency will be the rotor speed.

There is more than one way to calculate the AF for a given system. From unbalance response data, the half-power method is used. The half-power method equation is shown in Eqn. (7), where $N_{c}$ is the critical speed and $N_{2,1}$ are the speeds on either side of the critical at which the amplitudes are the critical speed amplitude to the $1 / 2$ power. 


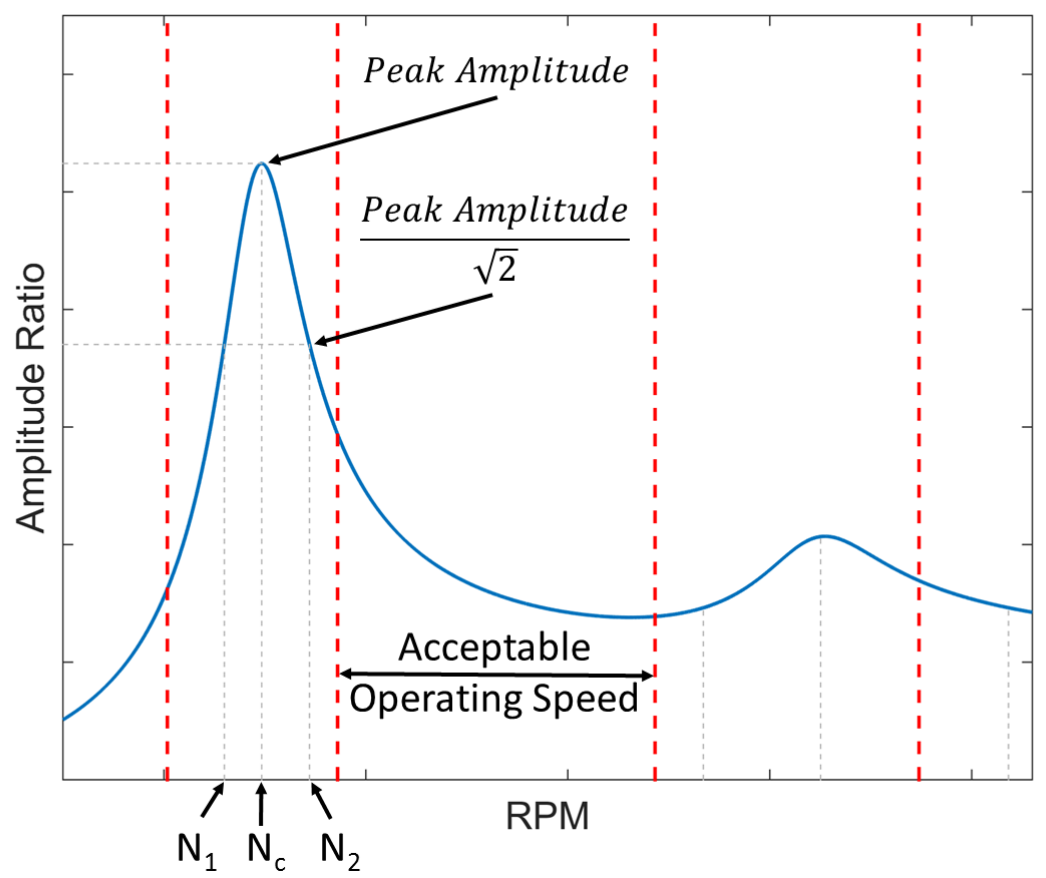

Fig. 19: An unbalance response which shows two critical speeds and their required separation margins

$$
A F=\frac{N_{c}}{N_{2}-N_{1}}
$$

For the plot in Fig. 19, the first critical speed will have a higher AF than the second critical speed as its shape is much narrower, denoting a higher sensitivity to a change in speed.

The amplification factor can also be calculated in terms of the modal damping ratio. The modal damping ratio is calculated from the damped eigenvalues of the system. Each of the two critical speeds seen in Fig. 19 will have a modal damping ratio. The method used to calculate the $\mathrm{AF}$ from the modal damping ratio is given by Eqn. (8), where $\zeta_{\text {modal }}$ is the damping ratio of the mode in question.

$$
A F=\frac{1}{2 \zeta_{\text {modal }}}
$$

The amplification factors calculated by these two methods can result in consider- 
ably different values. The unbalance amplitude and phase plots (often referred to as Bode plots) are typically the only data gathered from a machine in the field. Therefore the half-power method is used to calculate the AF in these cases. When a full computational rotor model is available, the modal damping ratios can be obtained and the AF can be calculated using Eqn. (8).

The differences in the two calculation methods can be related back to the damped eigenvalues and unbalance response of a single degree of freedom model. For low values of damping, the amplification factors calculated from Eqn. 7 and Eqn. 8 result in similar values. The damped natural frequency, however, is lower than the critical speed calculated by the unbalance response due to damping and the forcing function (Eqn. 5). An advantage of using the damped eigenvalues for calculation of the amplification factors is that, for very complex rotors, the damped eigenvalues are the same for the entire system whereas the amplification factors calculated from the unbalance response can change along different stations in the rotor. It is important to maintain consistency between the two methods when comparing the amplification factors from various data.

\section{Separation Margin}

The two pairs of heavy dotted vertical lines depicted in Fig. 19 are referred to as separation margins. Separation margins are required for any critical speed with an amplification factor greater than 2.5 (API $6842^{\text {nd }}$ Edition 2005). Looking at the first critical speed, for example, the two lines on either side of the critical peak show the speeds outside of which it is safe to operate. The separation margins are functions of the amplification factor and the critical speed's relative location to the operating speed. If the critical speed is below the minimum operating speed, the separation

margin (as a percentage of the minimum operating speed) is the lesser of 16 and the 
value defined by Eqn. (9).

$$
S M=17\left(1-\frac{1}{A F-1.5}\right)
$$

If the critical speed is above the maximum operating speed, the separation margin (as a percentage of the maximum operating speed) is the lesser of 26 and the value defined by Eqn. (10).

$$
S M=10+17\left(1-\frac{1}{A F-1.5}\right)
$$

For example, take the unbalance response data shown in Fig. 20. Given a minimum

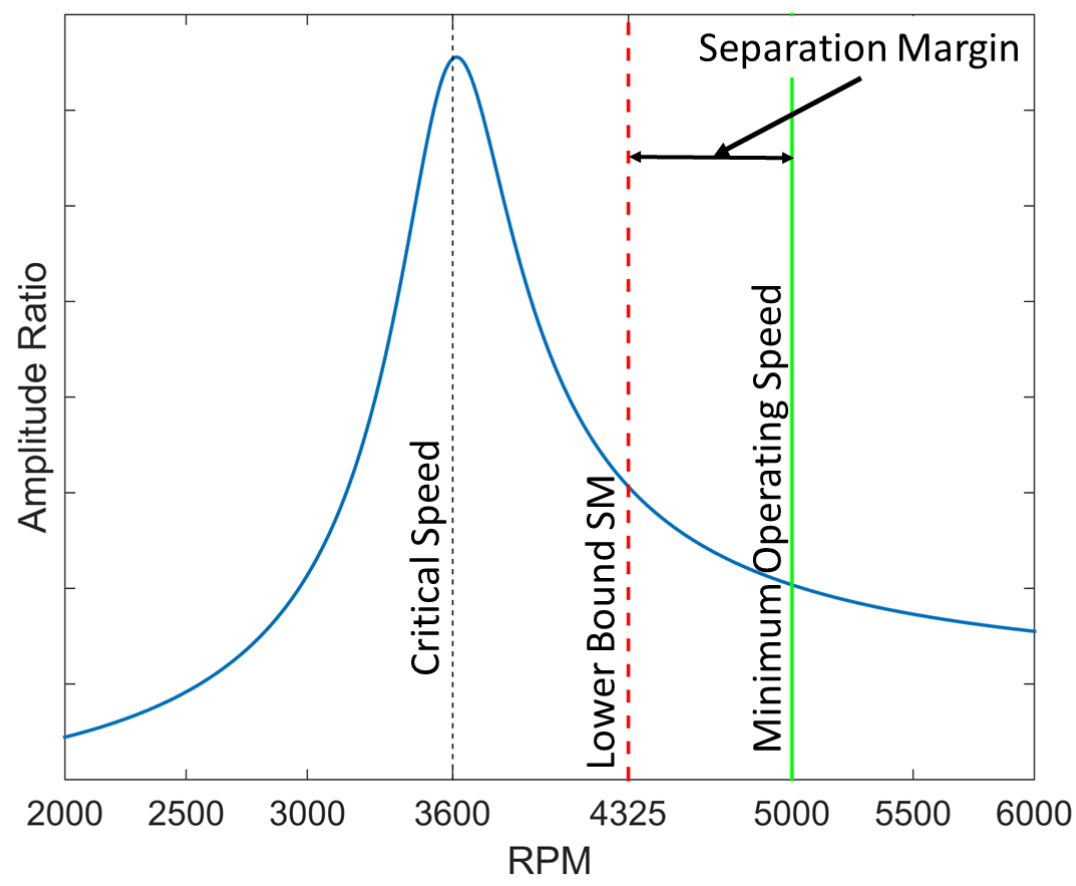

Fig. 20: An example of the first critical speed response due to unbalance. The red dotted line to the left of the green dotted line shows that this critical speed is at a safe distance from the operating speed and separaiton margin.

operating speed of 5,000 RPM, it can be seen that the critical speed (3,600 RPM with $\mathrm{AF}=7.3)$ is below the minimum operating speed and is therefore defined by Eqn. (9). 
Therefore, the separation margin speed can be calculated as:

$$
\begin{gathered}
S M=\left(17\left(1-\frac{1}{7.3-1.5}\right)\right)=13.5 \\
S M_{\text {bound }}=5,000-5,000(.135)=4,325 R P M
\end{gathered}
$$

In this case, it can be seen that the critical speed $(3,600 \mathrm{RPM})$ is below the required lower bound of the separation margin (4,325 RPM), and is therefore acceptable under the API standards.

It should be noted that if a critical speed has an amplification factor of less than 2.5, the response is considered critically damped and no separation margin is required under API specifications.

\section{Appendix B: Jeffcott rotor analysis}

The equations of motion for the Jeffcott rotor are coupled by the shared bearings and supports which are included in both modes (Fig. 21). The equation of motion for the Jeffcott rotor is given by Eqn. 11 where $x$ is the vector of degrees of freedom (Eqn. 12). This model was used to generate Fig. 5 and Fig. 6.

$$
[M] \ddot{x}+[C] \dot{x}+[K] x=0
$$




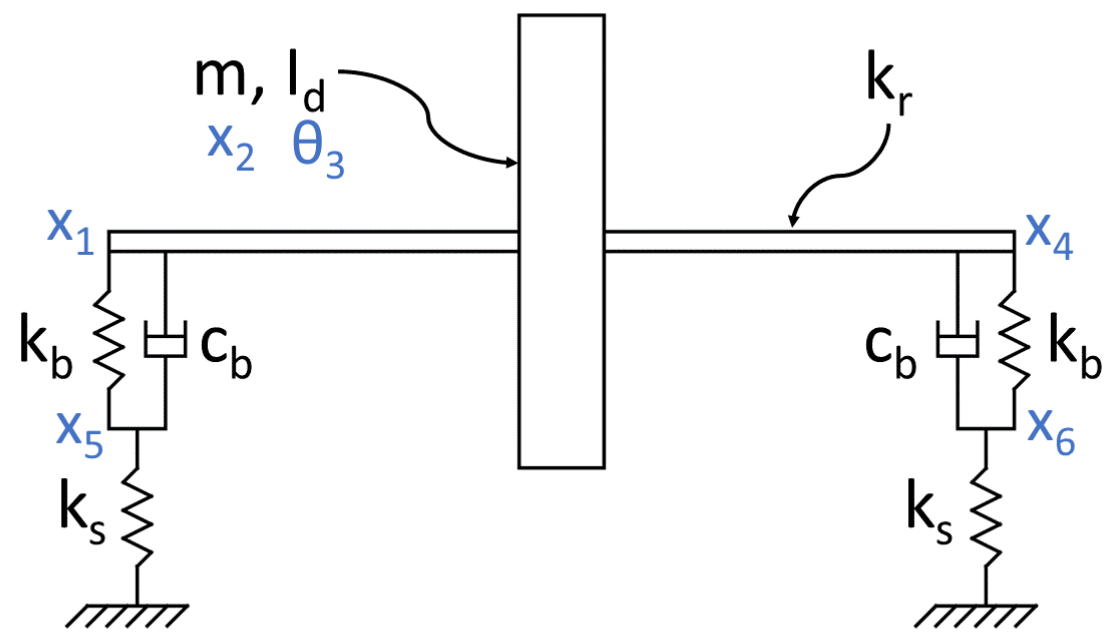

Fig. 21: The Jeffcott rotor model with degrees of freedom labeled

$$
\vec{x}=\left[\begin{array}{l}
x_{1} \\
x_{2} \\
\theta_{3} \\
x_{4} \\
x_{5} \\
x_{6}
\end{array}\right]
$$

For the rigid-support, rigid-bearing Jeffcott rotor modes, the lateral and rotational rotor stiffness are related by Eqns. 13-15.

$$
k_{r}=\frac{48 E I}{\ell^{3}}
$$




$$
\begin{gathered}
k_{\theta}=\frac{12 E I}{\ell} \\
k_{\theta}=\frac{k_{r} \ell^{2}}{4}
\end{gathered}
$$

The rotational motion for the second degree of freedom is converted to translational motion by Eqn. 16 .

$$
\theta=\frac{x}{\ell}
$$

The mass, stiffness, and damping matrices in terms of lateral motion are shown below:

$$
[M]=\left[\begin{array}{cccccc}
m_{1} & 0 & 0 & 0 & 0 & 0 \\
0 & m_{2} & 0 & 0 & 0 & 0 \\
0 & 0 & I_{d} & 0 & 0 & 0 \\
0 & 0 & 0 & m_{4} & 0 & 0 \\
0 & 0 & 0 & 0 & m_{5} & 0 \\
0 & 0 & 0 & 0 & 0 & m_{6}
\end{array}\right]
$$




$$
[K]=\left[\begin{array}{cccccc}
k_{b}+\frac{k_{r}}{2} & -\frac{k_{r}}{2} & \frac{k_{r}}{4} & 0 & -k_{b} & 0 \\
-\frac{k_{r}}{2} & k_{r} & 0 & -\frac{k_{r}}{2} & 0 & 0 \\
\frac{k_{r}}{4} & 0 & \frac{k_{r}}{4} & -\frac{k_{r}}{4} & 0 & 0 \\
0 & -\frac{k_{r}}{2} & -\frac{k_{r}}{4} & k_{b}+\frac{k_{r}}{2} & 0 & -k_{b} \\
-k_{b} & 0 & 0 & 0 & k_{b}+k_{s} & 0 \\
0 & 0 & 0 & -k_{b} & 0 & k_{b}+k_{s}
\end{array}\right]
$$

$$
[C]=\left[\begin{array}{cccccc}
c_{b} & 0 & 0 & 0 & -c_{b} & 0 \\
0 & 0 & 0 & 0 & 0 & 0 \\
0 & 0 & 0 & 0 & 0 & 0 \\
0 & 0 & 0 & c_{b} & 0 & -c_{b} \\
-c_{b} & 0 & 0 & 0 & c_{b} & 0 \\
0 & 0 & 0 & -c_{b} & 0 & c_{b}
\end{array}\right]
$$

Non-dimensionalizing the matrices by $k_{r}$ results in:

$$
[M]=\left[\begin{array}{cccccc}
\frac{\alpha}{\omega_{n 1}} & 0 & 0 & 0 & 0 & 0 \\
0 & \frac{1}{\omega_{n 1}} & 0 & 0 & 0 & 0 \\
0 & 0 & \frac{1}{4(M F R)^{2} \omega_{n 1}^{2}} & 0 & 0 & 0 \\
0 & 0 & 0 & \frac{\alpha}{\omega_{n 1}} & 0 & 0 \\
0 & 0 & 0 & 0 & \frac{\alpha}{\omega_{n 1}} & 0 \\
0 & 0 & 0 & 0 & 0 & \frac{\alpha}{\omega_{n 1}}
\end{array}\right]
$$




$$
[K]=\left[\begin{array}{cccccc}
\frac{R S R+1}{2} & -\frac{1}{2} & \frac{1}{4} & 0 & -\frac{R S R}{2} & 0 \\
-\frac{1}{2} & 1 & 0 & -\frac{1}{2} & 0 & 0 \\
\frac{1}{4} & 0 & \frac{1}{4} & -\frac{1}{4} & 0 & 0 \\
0 & -\frac{1}{2} & -\frac{1}{4} & \frac{R S R+1}{2} & 0 & -\frac{R S R}{2} \\
-\frac{R S R}{2} & 0 & 0 & 0 & \frac{(1+K) R S R}{2} & 0 \\
0 & 0 & 0 & -\frac{R S R}{2} & 0 & \frac{(1+K) R S R}{2}
\end{array}\right]
$$

Where $\alpha$ is a factor $(\alpha \approx 0)$ used to approximate a massless node, and $M F R$ is the mode frequency ratio given by Eqn. $17 . \omega_{n 1}$ and $\omega_{n 2}$ refer to the first and second rigid bearing natural frequencies respectively.

$$
M F R=\frac{\omega_{n 2}}{\omega_{n 1}}
$$

To find the damped eigenvalues of the system, a state-space formulation was used (Eqn. 18) and solved using Matlab (Eqn. 19). 


$$
[A]=\left[\begin{array}{cc}
{[0]} & {[I]} \\
-[M]^{-1}[K] & -[M]^{-1}[C]
\end{array}\right]
$$

$$
[D]=\operatorname{eig}([A])
$$

For mode frequency ratios $(M F R)$ above approximately 2 , there is little change in the equivalent parameters as listed in Tab. 1. Because of this, the Jeffcott rotor model may be simplified into the modal model shown in Fig. 22. This model was used to generate Fig. 7-10.

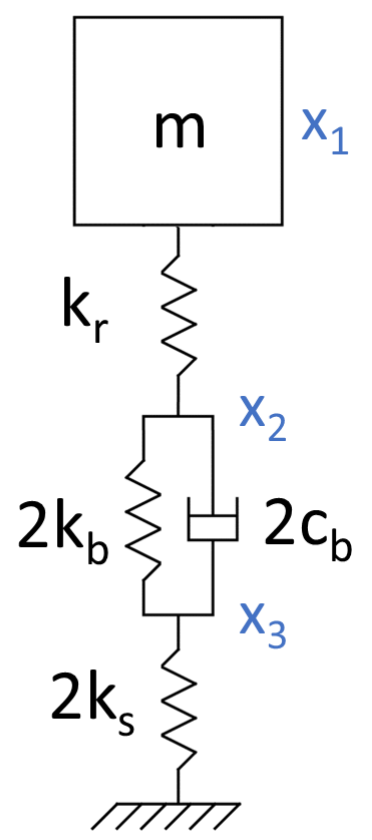

Fig. 22: The reduced Jeffcott rotor model with degrees of freedom labeled

The non-dimensionalized matrices for the reduced Jeffcott model are shown below. 
Using this method, the inputs for RSR and $\zeta$ should be based on modal values (from a rotordynamics software package) if this analysis is to be related to actual machine parameters.

$$
\begin{gathered}
{[M]=\left[\begin{array}{ccc}
\frac{1}{\omega_{n 1}{ }^{2}} & 0 & 0 \\
0 & \frac{\alpha}{\omega_{n 1}{ }^{2}} & 0 \\
0 & 0 & \frac{\alpha}{\omega_{n 1}{ }^{2}}
\end{array}\right]} \\
{[K]=\left[\begin{array}{ccc}
1 & -1 & 0 \\
-1 & R S R+1 & -R S R \\
0 & -R S R & (1+K) R S R
\end{array}\right]} \\
{[C]=\left[\begin{array}{ccc}
0 & 0 & 0 \\
0 & \frac{2 \zeta \sqrt{R S R}}{\omega_{n 1}} & -\frac{2 \zeta \sqrt{R S R}}{\omega_{n 1}} \\
0 & -\frac{2 \zeta \sqrt{R S R}}{\omega_{n 1}} & \frac{2 \zeta \sqrt{R S R}}{\omega_{n 1}}
\end{array}\right]}
\end{gathered}
$$

\section{Appendix C: Influence of damping}

The influence of support damping will be demonstrated using the steam turbine case study from Section 5. Typically 1-10\% modal damping is typical of a supporting structure; however, since the support in this analysis is massless, a percentage of the bearing damping is used (J. Nicholas and L. Barrett 1986). Figure 23 shows the second mode unbalance response of the steam turbine with no damping and with $10 \%$ of the bearing damping. Even with $10 \%$ of the bearing damping (a relatively large amount of damping), there is only negligible response change. Figure 24 shows 
a portion of the separation margin map for the second forward mode of the steam turbine with and without $10 \%$ support damping. Again, there is negligible change in the position of the mode.

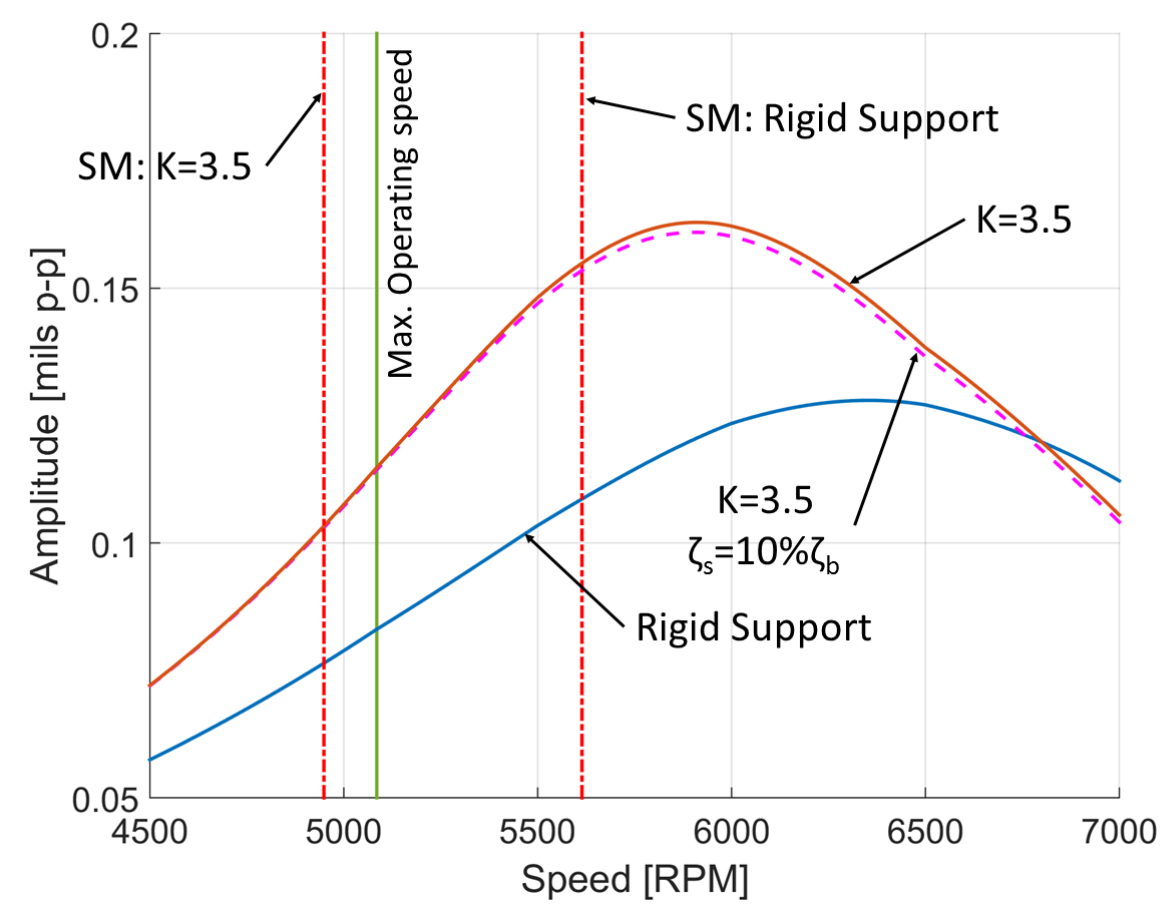

Fig. 23: The second mode unbalance response of the steam turbine with and without $10 \%$ support damping 


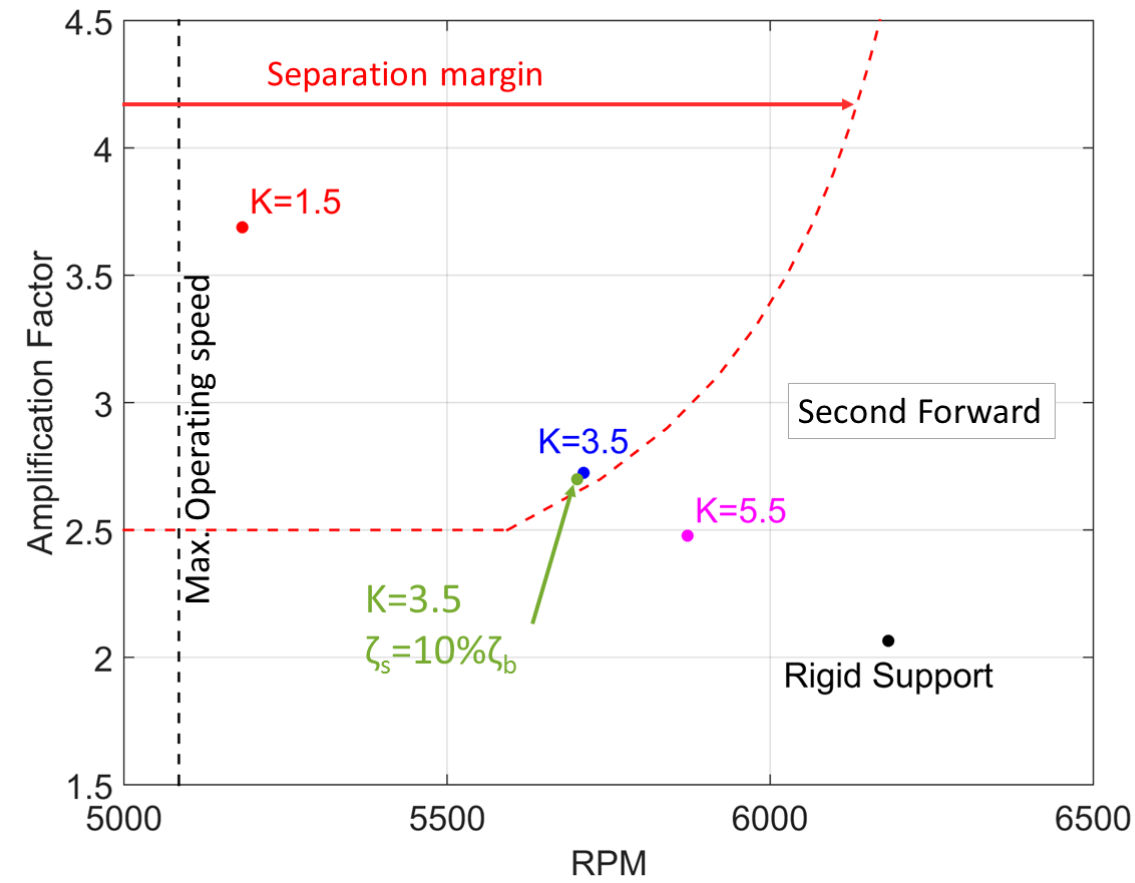

Fig. 24: The portion of the separation margin map for the second forward mode of the steam turbine with and without $10 \%$ support damping 